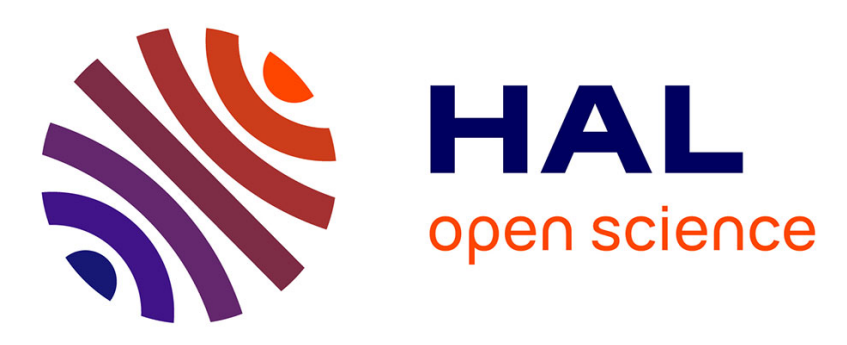

\title{
Pt-ttpy, a G-quadruplex binding platinum complex, induces telomere dysfunction and G-rich regions DNA damage
}

Samar Ali, Emilia Puig Lombardi, Deepanjan Ghosh, Tao Jia, Géraldine Vitry, Lina Saker, Joël Poupon, Marie-Paule Teulade-Fichou, Alain Nicolas, Arturo Londono-Vallejo, et al.

\section{To cite this version:}

Samar Ali, Emilia Puig Lombardi, Deepanjan Ghosh, Tao Jia, Géraldine Vitry, et al.. Pt-ttpy, a Gquadruplex binding platinum complex, induces telomere dysfunction and G-rich regions DNA damage. Metallomics, 2021, 10.1093/mtomcs/mfab029 . hal-03326341

\section{HAL Id: hal-03326341 https://hal.science/hal-03326341}

Submitted on 25 Aug 2021

HAL is a multi-disciplinary open access archive for the deposit and dissemination of scientific research documents, whether they are published or not. The documents may come from teaching and research institutions in France or abroad, or from public or private research centers.
L'archive ouverte pluridisciplinaire HAL, est destinée au dépôt et à la diffusion de documents scientifiques de niveau recherche, publiés ou non, émanant des établissements d'enseignement et de recherche français ou étrangers, des laboratoires publics ou privés. 
Metallomics 2021, 13, 6, mfab029

\section{Pt-ttpy, a G-quadruplex binding platinum complex, induces telomere dysfunction and G-rich regions DNA damage}

Samar Ali ${ }^{1}$, Emilia Puig Lombardi' ${ }^{2}$, Deepanjan Ghosh ${ }^{3,4}$, Tao Jia ${ }^{3,4}$, Géraldine Vitry ${ }^{1}$, Lina Saker ${ }^{1}$, Joël Poupon $^{5}$, Marie-Paule Teulade-Fichou ${ }^{3,4}$, Alain Nicolas ${ }^{2}$, Arturo Londono-Vallejo ${ }^{2}$ and Sophie Bombard $^{3,4^{*}}$

1, INSERM UMRS 1007, Université de Paris, 75006 Paris

${ }^{2}$ Institut Curie, PSL Research University, CNRS UMR3244, Sorbonne Université, Telomeres and Cancer lab, 75005 Paris

${ }^{3}$ Institut Curie, CNRS UMR9187-INSERMU1196, CMBC, 91405 Orsay, France

${ }^{4}$ Institut Curie, CNRS UMR9187-INSERMU1196, Université Paris-Saclay, 91405 Orsay, France

${ }^{5}$ Hôpital Lariboisière, Laboratoire de Toxicologie Biologique, 2 rue Ambroise Paré, 75475 Paris, France

*Corresponding author : sophie.bombard@curie.fr

Running title: Anti-cancer research of Pt-ttpy 


\begin{abstract}
Pt-ttpy (tolyl terpyridin-Pt complex) covalently binds to G-quadruplex (G4) structures in vitro and to telomeres in cellulo via its Pt moiety. Here, we identified its targets in the human genome, in comparison to Pt-tpy, its derivative without G4 affinity, and cisplatin. Pt-ttpy, but not Pt-tpy, induces the release of the shelterin protein TRF2 from telomeres concomitantly to the formation of DNA damage foci at telomeres but also at other chromosomal locations. $\gamma-\mathrm{H} 2 \mathrm{AX}$ chromatin immunoprecipitation (ChIP-seq) after treatment with Pt-ttpy or cisplatin revealed accumulation in Gand A-rich tandemly repeated sequences, but not particularly in potential G4 forming sequences. Collectively, Pt-ttpy presents dual targeting efficiency on DNA, by inducing telomere dysfunction and genomic DNA damage at specific loci.
\end{abstract}

\title{
Graphical abstract
}

Dual targeting of Pt-ttpy at telomeres and G-rich sequences

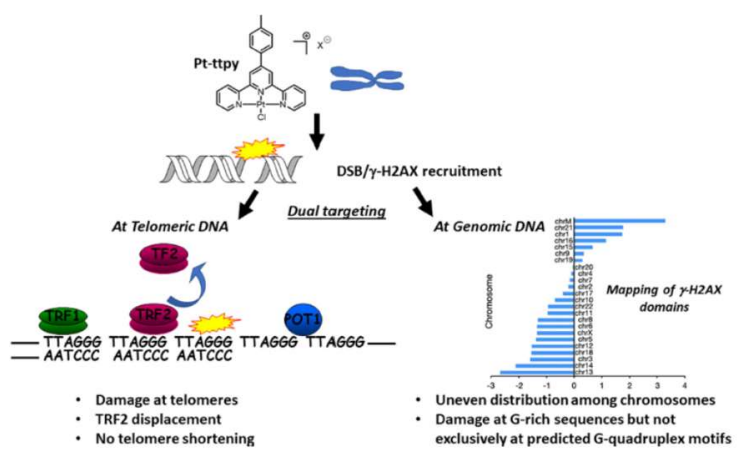

\section{Key words}

G-quadruplex, platinum complexes, telomere dysfunction, DNA damage, ChIP seq, TRF2 


\section{Introduction}

G-quadruplexes (G4) are stable nucleic acid secondary structures that are formed in DNA and RNA containing several G-runs [1]. They consist of the stacking of G-tetrads stabilized by the presence of physiological concentrations of monovalent cations. Substantial evidence shows that G4 DNA and RNA structures form in vivo. Bioinformatics analyses have predicted that the human genome contains 350,000 to 650,000 potential G4 forming sequences [2-4]. 700,000 G4s have been identified from DNA cellular extracts using in vitro polymerase stop assay [5]. However, in cellular context, BG4 ChIP-seq experiments have led to the identification of $10,000 \mathrm{G} 4 \mathrm{~s}$ in HaCaT cells, where they have been shown to be mainly located in regulatory regions of DNA [6]. These were unevenly distributed and mostly found in promoter of genes, particularly those of several oncogenes. While G4s are stable in solution, they consist in dynamic structures in cells due to associated proteins, supercoiling conditions, cell cycle state $[7,8]$. In addition, G4 formation has been intensively studied at telomeres, which are particularly predisposed to form such structures due to their G-richness organized in TTAGGG tandem repeats [9, 10]. Telomeres constitute the essential and specialized nucleoprotein structure that is located at the end of chromosomes and functions as a specialized DNA "cap", protecting chromosome ends from degradation and eliciting DNA repair activities [11]. A complex of six proteins called shelterin ensures the protection of telomeres [12]. Among them, TRF1 and TRF2 (telomeric repeat binding factors 1 and 2) and POT1 (Protection of Telomere 1) are directly bound to telomeric DNA. Telomere uncapping by release of TRF2 or POT1 induces telomere dysfunction and cell senescence or death [13], thus making telomeric DNA and its shelterin promising targets for anticancer therapy [14]. In all, it is well established that G4s play important roles in a broad range of biological processes $[15,16]$, including telomere maintenance [17], replication [18], transcription and translation [19]. Therefore, a large number of G4-interacting molecules [20, 21], including metallic complexes[22, 23], have been developed and many of them have been shown to reduce cancer growth [24]. Some of them such as CM03 or Quarfloxin have shown great potential for clinical use for resistant cancerous cell lines and revealed the down-regulation of a large number of genes which are rich in putative G-quadruplex forming sequences involved in essential survival pathways or rRNA biogenesis $[25,26]$

G4-interacting platinum complexes bearing a leaving group (typically $\mathrm{Cl}^{-}$or $\mathrm{I}^{-}$) are able to bind covalently in vitro to $\mathrm{G} 4 \mathrm{~s}$ by coordinating $\mathrm{N}_{7}$ or $\mathrm{N}_{1}$ of adenines or $\mathrm{N}_{7}$ of guanines [27-32]. Among these, the tolyl-terpyridin-platinum complex (Pt-ttpy) (scheme 1) preferentially stabilizes G4s in vitro with regard to duplex DNA $[33,34]$ by stacking to external G-tetrads [35] and is also able to efficiently trap G4s covalently by direct coordination to loop bases [30, 35]. Pt-ttpy displays potent anti-tumor activity [34] and one of its derivatives, Pt-ctpy, was shown to have promising radiosensitizing properties [36]. In line with its potential G4-sequence targeting ability, Pt-ttpy has been shown to bind covalently to 
telomeric DNA in cellulo [37], and to induce chromosome loss and ultrafine bridges formation that may be explained by telomere dysfunctions [38].

Since Pt-ttpy combines G4 stabilization, metal complex and direct DNA-metal coordination properties, it appears of great interest to identify its molecular targets and decipher its mechanism of action. We tested whether the biological activity of Pt-ttpy could be related to its G4-binding properties, using as controls two platinum complexes with poor or no affinity for G4s, the terpyridineplatinum complex Pt-tpy [34] and the anticancer drug cisplatin (Scheme 1). Pt-ttpy and Pt-tpy form monofunctional DNA adducts [30], whereas cisplatin forms mainly bifunctional DNA adducts between two adjacent guanines (1,2 intrastrand cross-links) [39]. Here, we show that Pt-ttpy appears as a more potent agent than cisplatin, as it is able i) to overcome cisplatin resistance through increased cellular uptake, ii) to target telomeres by inducing telomeric DNA damage and telomere deprotection and iii) to target genomic DNA by inducing DNA damage preferentially at G- and A-rich regions. We also show that partial TRF2 displacement from telomeres by drug treatment is not sufficient to induce telomeric DNA damage. Moreover, the analysis of the DNA damage sites induced by cisplatin treatment, through Chromatin-Immunoprecipitation sequencing, indicates that these are not correlated to the primary binding site if this platinum complex but rather reflect the persistent DNA damage sites distribution. This finding might also extend to Pt-ttpy and more largely to DNA-interactive Pt-complexes.
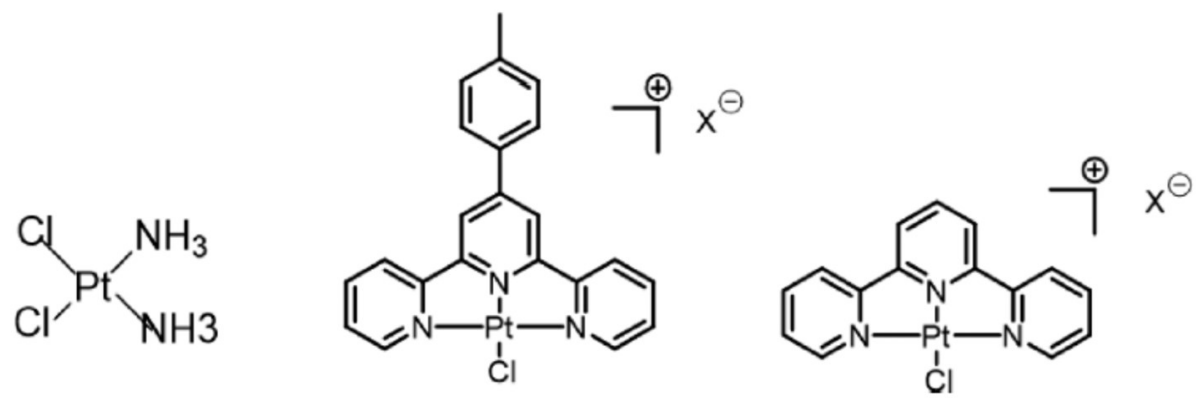

Scheme 1 Chemical structure of cisplatin, Pt-ttpy, and Pt-tpy. cellular extracts using in vitro polymerase stop assay. 5 However, in cellular context, BG4 Chromatin Immunoprecipitation

\section{Material and Methods}

\section{Cell culture.}

The ovarian carcinoma cell lines were purchased from ECACC (number 93112519 for A2780 and 93112517 for A2780cis, Salisbury, UK) and were grown in complete RPMI medium supplemented with $10 \%$ fetal calf serum, in the presence of $1 \%$ penicillin and streptomycin. The resistance of A2780cis cells to cisplatin was maintained by monthly treatment with $1 \mu \mathrm{M}$ cisplatin for 4 days. Cells were 
treated with various concentrations of Pt-ttpy, Pt-tpy and cisplatin at $37^{\circ} \mathrm{C}$ under humidity and $5 \% \mathrm{CO}_{2}$ conditions for $96 \mathrm{~h}$. Cellular growth was quantified using the particle counter Z2 Coulter', (Beckman, COULTER ${ }^{\circ}$ ). For growth inhibition cells were seeded in 6-well plates (100 000 cells) and allowed to attach for $6 \mathrm{~h}$. Cells were then treated with the indicated concentrations of the complexes and incubated for $96 \mathrm{~h}$. Cells were then trypsined and reseeded in an adequate volume of culture medium and counted with a cell counter (Coulter Z2, Beckman or MOXI Z Mini Automated Cell Counter, Orflo). We favored cell counting over the commonly tests used to determine cell proliferation (MTT, MTS, Titer-Glo...) because we found some discrepancies, as other groups [40], between these methods based on mitochondrial functions and manual cell counting. Indeed, we suspect that Pt-ttpy effects on mitochondria homeostasis biased the results of cell viability given by MTT, MTS, WST-1 and Titer-Glo assays. The Cell Viability was performed on the Cellometer Auto 2000 Cell Viability Counter (Nexcelom) using the dual AO/PI labelling which provides a convenient pre-mixed AO/PI solution that can be directly added to cell samples for cell counting and viability analysis

\section{Platinum complexes}

Cisplatin was provided from Sigma. Pt-ttpy and Pt-tpy were synthesized following the procedure already described [34]. Aqueous solutions of cisplatin $1 \mathrm{mM}$, of Pt-tpy $1 \mathrm{mM}$ and $6 \mathrm{mM}$ DMSO solutions of Pt-ttpy were prepared and conserved at $-20^{\circ} \mathrm{C}$. Of note, the Pt-ctpy derivative was not chosen because this derivative has a lower G4-selectivity in vitro as compared to Pt-ttpy [34]. Diluted solutions of each molecule were freshly prepared.

\section{Platinum uptake measurement}

The platinum cellular uptake was quantified by ICP-MS (inductively coupled plasma mass spectrometry, NexION 2000, Perkin Elmer) on cellular pellet ( $5 \times 10^{6}$ cells), DNA extract (using DNeasy kit from Qiagen) as previously described [41] in A2780 or A2780cis cells treated with various concentrations of Pt-ttpy, Pt-tpy and cisplatin during indicated time treatment Around 20-25 $\mathrm{g}$ DNA (quantified by nanodrop) were extracted from pellets of $5 \times 10^{6}$ cells. ICP-MS has been used also for monitoring the solubilization of platinum complexes in the aqueous solution. Prior ICP-MS, the samples were digested with PlasmaPURE Plus $\mathrm{HNO}_{3}(69 \%)$ at $95^{\circ} \mathrm{C}$ for cell pellets and mitochondria and $\mathrm{HNO}_{3}$ 0.1M for DNA. The amount of Pt content was determined following a dose response curve established from known concentrations of cisplatin. The amount of platinum was then reported as $\mathrm{ng}$ of $\mathrm{Pt} / 5 \times 10^{6}$ cells or pg Pt/ug DNA

\section{Annexin V Apoptosis Assay}


The $A 2780$ cells were plated in a 6 well plate $\left(100000\right.$ cells) with the $I C_{80}$ concentration of the drug. After 96 hours of treatment. the FITC Annexin V Apoptosis Detection Kit I (BD Pharmingen) was used to label the cells. The cells are washed with PBS and suspended in 1X binding buffer at a concentration of $1 \times 10^{6} \mathrm{cells} / \mathrm{ml}$. $100 \mu \mathrm{l}$ was transferred to a $5 \mathrm{ml}$ culture tube and $5 \mu \mathrm{l}$ of FITC Annexin V and $5 \mu \mathrm{l} \mathrm{PI}$ were added. The cells were gently vortexed and incubated at RT for 15 minutes in the dark. After addition of $400 \mu \mathrm{l}$ of $1 \mathrm{X}$ binding buffer to each tube. Flow cytometry acquisitions were obtained on a FACSCanto $\mathrm{II}^{\mathrm{TM}}$ analyzer (BD Biosciences) with the assistance of BD FACSDiva Software (BD Biosciences) and data analyzed with the FlowJo Software (Tree Star).

\section{ChIP assay for detection of TRF2, TRF1, POT1 and H3 binding}

ChIP was carried out using a Chromatin IP (ChIP) assay kit according to the manufacturer's instructions (Upstate). Cells were collected after fixation of proteins with formaldehyde, and lysed. DNA of nucleus was sonicated until fragments of $1 \mathrm{kbp}$ were obtained. $30 \mu \mathrm{l}$ was conserved in order to quantify the number of telomeric sequences before immuno-precipitation (INPUT). Immunoprecipitation was then performed with anti-TRF2 polyclonal antibody (IMG-148A, IMGENEX), anti-TRF1 polyclonal antibody (ab1423, Abcam), anti-POT1 antibody, anti-histone H3 antibody (anti-H3, Abcam), or anti-lgG rabbit antibody (sc-2027, Abcam). 150ng of the immunoprecipitated DNA and from INPUT were blotted onto a Hybond-XL membrane (Ge HealthCare). The telomere sequences were detected using a 800bp telomere repeat (TTAGGG) ${ }^{32} \mathrm{P}$ labelled probe obtained after digestion of the pUC Telo2 plasmid [42] by EcoRI and BamHI and radiolabelled by random priming using dCTP [ $\left.\alpha^{32} P\right]$, TAGGGTTA/TAACCCTA (Eurogentec) as primers and Klenow polymerase (Fermentas). The Alu sequences were detected using a ${ }^{32} \mathrm{P}$ labelled Alu probe that was obtained after the digestion of the pTopo Alu-All plasmid (obtained after amplification of human genomic DNA with tgaaaccccgtctctactaaaaa and gtctcgctctgtcgccca primers, then cloned in PGEM-T vector (Promega)) by EcoRI and radiolabelled by random priming using $\mathrm{dCTP}\left[\alpha^{32} \mathrm{P}\right]$, the hexanucleotide mix (Roche) as primers and Klenow polymerase (Fermentas). The membranes were first hybridised with the telomere probe, and the amount of radioactivity was quantified using the Phosphorimager and ImageQuant software. The membranes were dehybridised in boiling water containing $1 \%$ SDS, and were then hybridised with the Alu probe; the amount of radioactivity was quantified using the Phosphorimager and ImageQuant software. Fold enrichment of the immunoprecipitated fraction compared to INPUT DNA is calculated as the ratio between telomeric DNA signals after precipitation and telomeric DNA signals in the total INPUT DNA for the same amount of blotted DNA (150ng). The values are normalised to the Alu signal in the immunoprecipitated and INPUT fractions for each condition using the (telomere IP/telomere INPUT)/(Alu IP/Alu INPUT) formula. 
The \% of TRF2 bound to telomeres was given as function of TRF2 bound in treated cells/TRF2 bound in untreated cells.

\section{PCR telomere length.}

Genomic DNA of A2780 treated cells with cisplatin, Pt-ttpy or Pt-tpy during four days at doses inducing 75\% growth inhibition was extracted using the DNeasy Blood \& Tissue Kit (QIAGEN) and the relative telomere length were determined by real-time PCR using the method described by Cawthon [43], adapted for a LightCycler instrument [44].

\section{Southern blot telomere length}

Genomic DNA was isolated from cells using the DNeasy ${ }^{\circ}$ blood and tissue Kit (Qiagen). Aliquot of $3 \mu \mathrm{g}$ DNA was digested overnight at $37^{\circ} \mathrm{C}$ with restriction enzymes Rsal and Hinfl. DNA fragments were separated by agarose gel electrophoresis, and then transferred under denaturing condition to a nylon membrane by Southern blotting. Telomere length was then estimated using the "Telo TAGGG Telomere Length Assay" kit (Roche).

\section{Western Blot}

Western blots were performed following Bio-Rad protocol. Briefly, $20 \mu \mathrm{g}$ proteins were electrophoresed in SDS-PAGE (SDS-Polyacrylamide 10\%) under denaturing conditions, then transferred to a PVDF membrane (Polyvinylidin Difluoride) (Amersham Hybond ${ }^{\mathrm{TM}} \mathrm{P}^{+}$, GE Healthcare) or nitrocellulose, which were hybridized with mouse monoclonal anti-TRF2 antibody (4A794, Upstate), the Anti-TRF2 Thr-188P polyclonal antibody and anti-actin HRP (SC1616-HRP, Santa-Cruz). TRF2 was revealed by the secondary antibody goat anti-mouse IgG-HRP (ab6789, abcam) using the ECL Western Blotting detection reagent. Western-blot membranes were analysed using FluorChem software program.

\section{Immunofluorescence Assays.}

A2780 cells plated on coverslips in 6-well plates. After 4 days of treatment cisplatin, Pt-ttpy or Pt-tpy during $96 \mathrm{~h}$ at doses inducing $80 \%$ growth inhibition, cells were washed with phosphate-buffered saline (PBS), then fixed 10 minutes in $4 \%$ formaldehyde. After a wash with PBS, cells were permeabilised 2 min using $0.5 \%$ Triton X-100 and washed with PBS. The cells were incubated in blocking buffer ( $5 \%$ bovine serum albumin in PBS) for 30 min before being incubated for $1 \mathrm{~h}$ with the primary mouse monoclonal antibody against TRF2 (clone 4A794), Anti-TRF2 Thr-188P polyclonal antibody [45], monoclonal mouse anti $\mathrm{Y}$-H2AX antibody (JBW301, Upstate), mouse monoclonal anti-TRF1 antibody (ab10579) or polyclonal rabbit anti $\mathrm{p}-\mathrm{H} 2 \mathrm{AX}$ antibody (ab2893) when used in co-localization with anti- 
TRF1 antibody for Telomere dysfunction-Induced Foci (TIF) experiments. After three washes with PBS, the cells were incubated for an additional $1 \mathrm{~h}$ with a goat anti-mouse IgG secondary antibody TRITC (tetramethylrhodamine)-conjugated (Thermofischer). Nuclei were counterstained with 4',6diamidino-2-phenylindole, Vectashield (DAPI). Three-dimension images (composed of 40 to 80 planes of $0.3 \mu \mathrm{m}$ ) were acquired using an inverted microscope with Epi-fluorescence attachment (Nikon Eclipse TE-2000 E) For co-localization experiments, nuclei were labeled using TO-PRO ${ }^{\circledR}-3$ (Life Technologies) and the coverslides were mounted with Vectashield ${ }^{\mathrm{TM}}$. Acquisitions were performed on confocal microscope (Zeiss LSM510) in the SCM (Faculté des Sciences Fondamentales et Biomédicales - Universite Paris Descartes). The number of foci in each nucleus was counted using the Image J software program after a two-dimensional projection of three-dimension images.

\section{Telomere dysfunction-Induced Foci (TIF) analysis}

Telo-FISH (Telomere-Fluorescence In Situ Hybridization) using peptide nucleic acid probes (PNA) conjugated to fluorescein isothiocyanate (CCCTAA)3-FITC (PANAGENE) was combined with immunofluorescence (IF) using a monoclonal mouse anti $\mathrm{Y}-\mathrm{H} 2 \mathrm{AX}$ antibody (JBW301, Upstate) in order to detect interphase telomere dysfunction-induced foci (TIF). A2780 cells plated on Chambered Coverglass (Lab-TeK, Nunc ${ }^{\mathrm{TM}}$ ) were fixed with $4 \%$ paraformaldehyde, then they were washed in PBS and dehydrated using ethanol at progressive concentrations (70\%, 80\%, 90\% and $100 \%) .5 \mu \mathrm{g} / \mathrm{ml}$ of PNA probe in hybridisation mixture containing $70 \%$ formamide, $10 \mathrm{mM}$ Tris- $\mathrm{HCl} \mathrm{pH} 7.2,1 \mathrm{mM} \mathrm{MgCl}$, $0.5 \%$ Boehringer blocking reagent and added to cells, followed by DNA denaturation for 2 min at $75^{\circ} \mathrm{C}$ and hybridisation for $1 \mathrm{~h} 30$ at room temperature. FISH protocol was followed by immunofluorescence staining, as previously described. Three-dimension images (composed of 25 planes of $0.8 \mu \mathrm{m}$ ) were acquired using an inverted confocal microscope (Axiovert 200M Zeiss LSM 510) in the SCM (Faculté des Sciences Fondamentales et Biomédicales - Université Paris Descartes). PNA foci, $\gamma$-H2AX foci and TIF, where PNA foci and $\mathrm{Y}-\mathrm{H} 2 \mathrm{AX}$ foci co-localised, were counted in each nucleus from a twodimensional projection of the three-dimension images using the JACoP plugin of Image J software program (NCBI).

\section{ChIP sample preparation}

The A2780 cells were treated with the $\mathrm{IC}_{80}$ doses of Pt-ttpy and cisplatin for 96 hours because under these conditions the two complexes induce $40-50 \%$ of damage to genomic DNA. At the end of the treatment, the cells were fixed with $4 \%$. After stopping the fixation by glycine $(0.125 \mathrm{M})$ the cells are recovered by scraping, centrifuged and lysed with the lysis buffer ( $5 \mathrm{mM}$ PIPES pH8, 85mM KCl, 0.5\% NP40, 1X inhibitor cocktail) using piston B (dounce homogenizer) 10 times in ice. The lysed cells are then aliquoted (approximately 2-4 million per tube) the nucleus was then lysed by the buffer ( $5 \mathrm{mM}$ 
Tris- $\mathrm{HCl}$ pH8, 10mM EDTA, 1\% SDS, $1 \mathrm{X}$ inhibitor cocktail) and sonicated to obtain fragments of size 200$700 \mathrm{bp}$. The sonicated chromatin was then incubated with ChIP Adembeads protein A / G (Sigma) which have already blocked for 15 minutes with $225 \mu$ l of "blocking buffer" and incubated with 1-3 $\mu \mathrm{g}$ of anti pH2AX antibody (anti -babbit ab2893) in IP buffer (0.01\% SDS, 1.1\% triton X-100, 1.2mM EDTA, 16.7mM Tris- $\mathrm{HCl}$ pH8, $16.7 \mathrm{mM} \mathrm{NaCl}$ ) The beads were then washed with several washing buffers which contain increasing concentrations of $\mathrm{NaCl}$, then the magnetic particles are suspended in $300 \mu$ l of elution buffer (0.1M NaHCO3, 1\% SDS) and proteinase K. DNA was recovered and purified using phenol / chloroform , and its concentration assayed with Nano-drop and qubit. Its quality was assayed by Agilent 2100 expert high sensitivity DNA assay and the library prepared and high-throughput sequencing done on HiSeq2500 (Rapid Flow cell,50 bases Single Read, Illumina TruSeq Read) by the NGS facility of the Institut Curie. The quality of the IP was also validated by contaminating samples with E. coli DNA which is only very weakly immunoprecipitated under these conditions.

\section{Library preparation and Sequencing}

Illumina compatible libraries were prepared from input and immunoprecipitated DNAs using the Illumina TruSeq ChIP library preparation kit according to the manufacturer's protocol. Briefly, 15ng of DNA were subjected to subsequent steps of end-repair, dA-tailing and ligation of TruSeq indexed Illumina adapters. After a final PCR amplification step, the 8 resulting barcoded libraries were equimolarly pooled and quantified using a qPCR method (KAPA library quantification kit, Roche) before sequencing on the Illumina HiSeq2500 instrument. The pool was loaded on 2 rapid flow cells and sequenced using a single read mode (read length of 100 bases, SR100). This sequencing configuration was set to reach an expected yield of 75 million reads per sample.

\section{ChIP-seq data analysis}

Mapping and peak calling. Raw fastq reads were aligned to the human reference genome (version hg19) using the BWA-mem algorithm (v0.7.5) [46]. We removed reads with a mapping quality inferior to ten or marked as positional duplicates. Peaks for ChIP experiments were detected using MACS2 (v.2.1.0) [47], for each replicate independently, with relevant input reads as background, an FDR threshold of 0.05 and default parameters. Peaks were also called for cisplatin and Pt-ttpy $\gamma$-H2AX IPs using the untreated condition reads as background. The obtained peaks were further filtered based on the condition fold change $>3$. Finally, we created the bed files used in all the analyses by keeping the peaks present at the intersection between replicates. These final bed files were imported into $\mathrm{R}$ for MacOSX [48] to generate the plots reported in the manuscript. 
Peak annotation and motif search. We used the annotatePeaks.pl Perl script from HOMER software (v4.9) [49] to annotate all the obtained peaks previously described and to calculate enrichments of the identified features. De novo and known motif discovery were carried out using the HOMER motif analysis pipeline. In addition, sequences within peak regions were scanned for for satlll repeats ([GGAAT $\left.]_{n}-\right)$, for telomere repeats $\left([T T A G G G]_{n}\right)$, canonical G-quadruplex motifs with up to 7 or 12 nucleotides in the loops $\left(\mathrm{G}_{3-5} \mathrm{~N}_{1-7} \mathrm{G}_{3-5} \mathrm{~N}_{1-7} \mathrm{G}_{3-5} \mathrm{~N}_{1-7} \mathrm{G}_{3-5}\right.$ or $\mathrm{G}_{3-5} \mathrm{~N}_{1-12} \mathrm{G}_{3-5} \mathrm{~N}_{1-12} \mathrm{G}_{3-5} \mathrm{~N}_{1-12} \mathrm{G}_{3-5}$ motifs), GG, GA or GNG occurrences by regular expression matching. The $\log _{2}$ fold-enrichments reported in the figures were calculated by comparing motif counts within peak regions to counts of the same peak regions after random shuffling of the sequences throughout the hg19 reference genome. Shuffling was performed with a Python implementation of the Altschul-Erikson dinucleotide shuffle algorithm. 


\section{Results}

\section{Antiproliferative properties of Pt-ttpy compared to Pt-tpy and cisplatin}

We examined the cell growth inhibition of Pt-ttpy and Pt-tpy for a 96h-treatment compared to cisplatin on the ovarian cell line A2780 and its counterpart cisplatin-resistant, A2780cis. This allowed determining the $\mathrm{IC}_{80}$, a dose that inhibits $80 \%$ proliferation, conditions that were used for the subsequent experiments. In A2780, the $\mathrm{IC}_{80}$ of Pt-ttpy and Pt-tpy (Figure 1 and Table 1) are similar (4 and $5.5 \mu \mathrm{M}$, respectively) but 7-fold higher than that of cisplatin $(0.66 \mu \mathrm{M})$, which is consistent with the reported $\mathrm{IC}_{50}$ values $\left(2.5,3\right.$ and $0.3 \mu \mathrm{M}$, respectively) [34]. To note, while the $\mathrm{IC}_{80}$ of Pt-ttpy is the same for A2780cis and A2780 cell lines and that of Pt-tpy is only slightly higher in A2780cis when compared to $A 2780$, the $I_{80}$ of cisplatin increases in $A 2780$ cis by a factor of 10 , as expected. These results indicate that the two terpyridine complexes show no (or almost no) cross-resistance with cisplatin (factor resistance of $1-1.7$, Table 1 ). Therefore, Pt-ttpy becomes more efficient than cisplatin (4 $\mu \mathrm{M}$ versus $7 \mu \mathrm{M})$ in A2780cis.
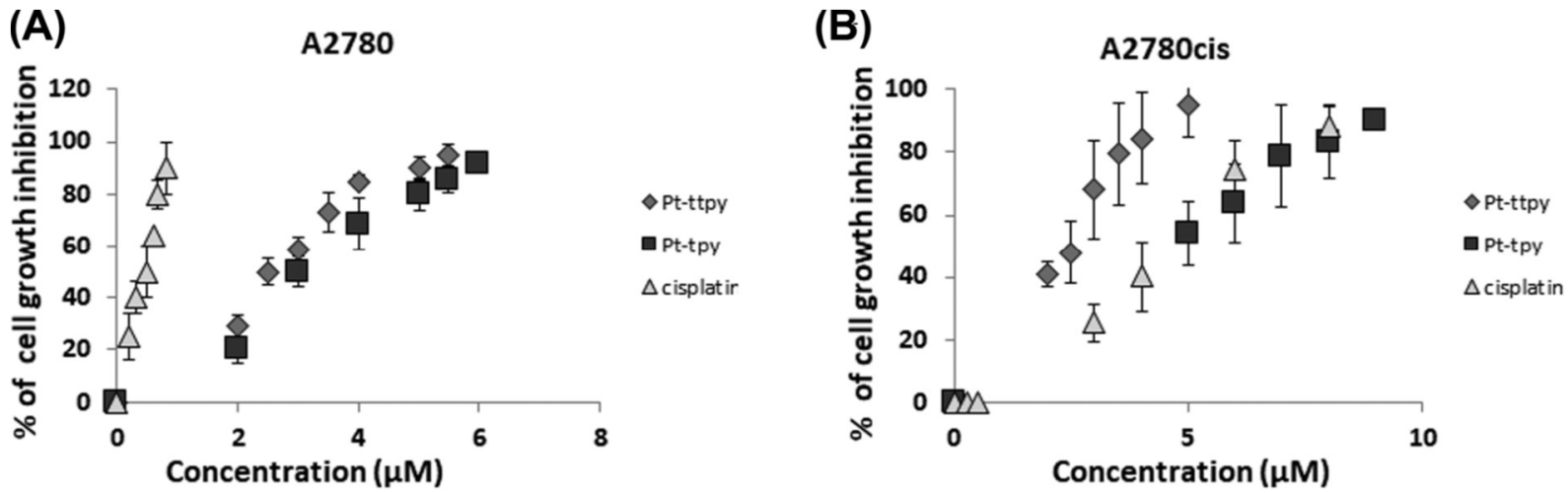

Fig. 1 Cell growth inhibition. (A) \% cell growth inhibition of A2780 cells by cisplatin, Pt-ttpy, and Pt-tpy. (B) \% cell growth inhibition of A2780cis cells by cisplatin, Pt-ttpy, and Pt-tpy. Cell growth was evaluated by cell counting using cell counter (At least 10 experiments).

\begin{tabular}{|c|c|c|c|c|c|c|}
\hline \multirow[b]{2}{*}{ Resitance factor $\left(\mathrm{IC}_{50}\right)$} & \multicolumn{3}{|c|}{ A2780 cells } & \multicolumn{3}{|c|}{ A2780cis cells } \\
\hline & Cis-Pt & Pt-ttpy & Pt-tpy & Cis-Pt 12.1 & Pt-ttpy 1 & Pt-tpy 1.7 \\
\hline $\mathrm{IC}_{50}(\mu \mathrm{M})$ & 0.33 & 2.5 & 3 & 4 & 2.5 & 5 \\
\hline Relative Uptake to cisplatin & 1 & 16 & 8 & 1 & 2.1 & 1.6 \\
\hline Relative DNA to cisplatin & 1 & 1.6 & 1.2 & 1 & 0.4 & 0.3 \\
\hline $\mathrm{IC}_{80}(\mu \mathrm{M})$ & 0.66 & 4 & 5.5 & 7 & 4 & 8 \\
\hline Relative Uptake to cisplatin & 1 & 9 & 7.4 & 1 & 1.4 & 1.5 \\
\hline Relative DNA to cisplatin & 1 & 0.8 & 0.7 & 1 & 0.4 & 0.3 \\
\hline
\end{tabular}

Table 1 IC50, IC80 of cisplatin, Pt-ttpy, and Pt-tpy in A2780 and A2780cis cells and their resistance factor (ratio IC50 A2780cis/IC50 A2780). Standard errors have been evaluated between 5 and 15\%. Relative values with respect to cisplatin of cellular uptake of Pt-tpy and Pt-ttpy (ng Pt for $5 \times 106$ cells) and platinum bound to DNA in A2780 and A2780cis cells (pg Pt per mg of genomic DNA) after treatment with cisplatin, Pt-ttpy, and Pt-tpy at their respective IC50 and IC80 (From Fig. 2). 


\section{Cellular uptake and genomic DNA binding of platinum complexes}

The discrepancy of the three complexes in anti-proliferative activity between both cell lines might result from their difference in cellular uptake and/or genomic DNA binding efficiency, since platinum DNA-adducts have been recognized as the ultimate event generated in cells $[39,50]$. At the stage, we want to point out that contrary to other platinum complexes, our complexes are not luminescent [51]. Therefore, to explore the cellular fate of the three platinum drugs, we determined their cellular uptake and distribution using the inductively-coupled plasma mass spectrometry (ICP-MS) method [52], which allows to quantify the amount of Pt in whole cells and that bound to genomic DNA. The cellular accumulation of the three complexes and their binding to DNA are time- and dose-dependent (Figure S1). For the 96h-treatment, the accumulation of both Pt-ttpy and Pt-tpy is stronger than that of cisplatin in A2780 cells ( 9 or 7.4 times more at $I C_{80}$, respectively, Figure2A and Table 1), however their binding to DNA is quite similar (Figure2B and Table 1). This suggests that anti-proliferative activity in A2780 cells is to be linked to their ability to bind DNA rather than to their cellular uptake. In A2780cis cells, as compared to A2780cells (Figure 2), cellular uptake and DNA binding are slightly increased for both Pt-ttpy and Pt-tpy, in contrast to cisplatin [53] where a large increase in uptake and DNA binding (7 times) due to their respective increase in drug concentration. These results are in agreement with those obtained when cells were treated at equimolar concentrations of drugs [37] and indicate that the influx/efflux of Pt-ttpy and Pt-tpy are not altered in A2780cis cells, contrary to cisplatin [54]. For cisplatin, both the cellular accumulation and amount bound to DNA in A2780cis cells are slightly higher than those reported in previous studies $[55,56]$, which may be attributed to different incubation conditions.

Given that all complexes show similar Pt-binding efficiency to DNA, we decided to explore their binding specificity for various DNA loci (telomeres, genomic G4 sequences) in conditions maximizing the formation of platinum adducts with DNA ( $96 \mathrm{~h} \mathrm{IC} 80$ doses). In order to explore these properties, we ensured of the viability of remaining adherent cells using the acridine orange/Propidium iodide viability assay after treatment with the drugs at $\mathrm{IC}_{80}$ for $96 \mathrm{~h}$ (Figure S2A). Further analysis by Annexin V-FITC/PI assay confirmed the absence of dead cells (late apoptotic cells) but evidenced an induction of early apoptotic cells restricted to Pt-ttpy treatment in these treatments' conditions (Figures S2B-C). 
(A)

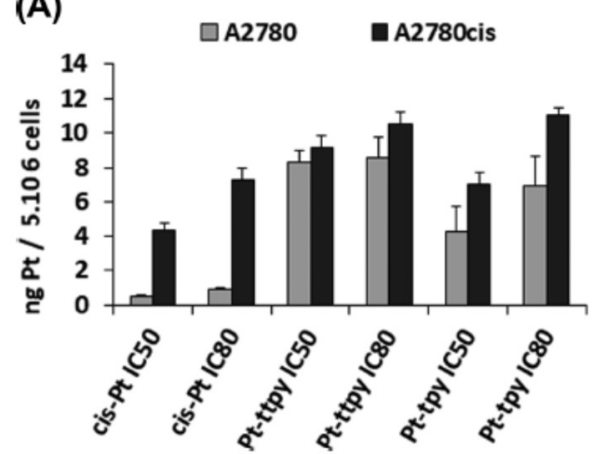

(B)

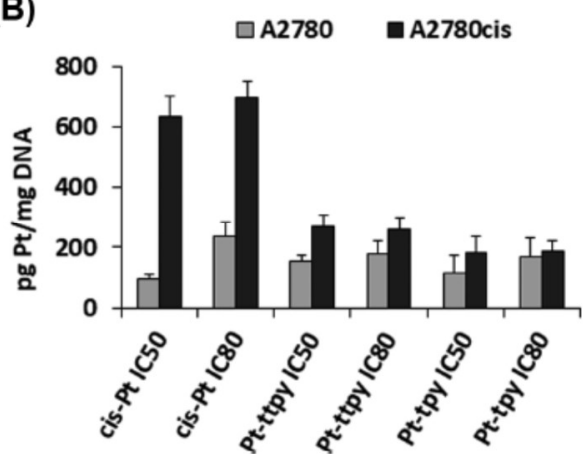

Fig.2: Cellular uptake and amount of platinum complexes bound to genomic DNA in A2780 and A2780cis cells. The metal content was determined after $96 \mathrm{~h}$ incubation time at the IC50 and IC80 doses of cisplatin (0.33 and $0.66 \mu \mathrm{M})$, Pt-ttpy (2.5 and $4 \mu \mathrm{M})$ and Pt-tpy ( 3 and $5.5 \mu \mathrm{M})$, respectively, in A2780 (grey bars) and of cisplatin ( 4 and $7 \mu \mathrm{M})$, Pt-ttpy $(2.5$ and $4 \mu \mathrm{M})$ and Pt-tpy ( 5 and $8 \mu \mathrm{M})$, respectively in A2780cis (black bars) for 5106 cells or rationalized per mg of genomic DNA extracted

Telomeric dysfunction is induced upon treatment with Pt-tty but not with Pt-tpy and cisplatin.

Since we have shown that Pt-ttpy and Pt-tpy may bind to telomeres by coordination, in an equal distribution on both telomeric and genomic DNA whereas a slight preference for genomic DNA for cisplatin was observed [37], we wondered if these binding events may affect the stability of telomeres and induce telomere dysfunction-induced foci (TIFs) [57]. Therefore, we evaluated the effects of the 3 platinum complexes on DNA damage response by $\gamma-\mathrm{H} 2 \mathrm{AX}$ immunostaining $(\gamma-\mathrm{H} 2 \mathrm{AX}$ foci), a well-defined marker of DNA damage. Cisplatin, Pt-ttpy and Pt-tpy induced a large amount of $\gamma$ H2AX foci formation (40-80 foci) (Figures $3 \mathrm{~A}, 3 \mathrm{~B}$ and S3A). Specific damage to telomeres was further assessed by the quantification of $\gamma-\mathrm{H} 2 \mathrm{AX}$ foci using a telomeric specific targeting PNA probe. Colocalisation analysis of the DNA damage signals with telomeres staining (Figure $3 \mathrm{~A}$ ) showed that only Pt-ttpy induced significant telomere damage (24\% of cells comprising 3-5 TIFs per cells) (Figure 3C). Since the binding of platinum complexes to telomeric DNA could partially impede the hybridization of telomeric probes to telomeres [37], Pt-ttpy-induced TIFs were confirmed by the co-localisation of the DNA damage signals with the telomeric protein TRF1 (Figure S3A), which is not released from telomeres upon the various treatments (as seen in Figure 4). As shown in Figure S3B, 35\% of cells treated by Pt-ttpy contain 3 to 8 telomeric damages per cell (average 2 TIFs per cell).

Therefore, among the three platinum complexes, only the G4-ligand, Pt-ttpy is able to induce telomeric DNA damage. 


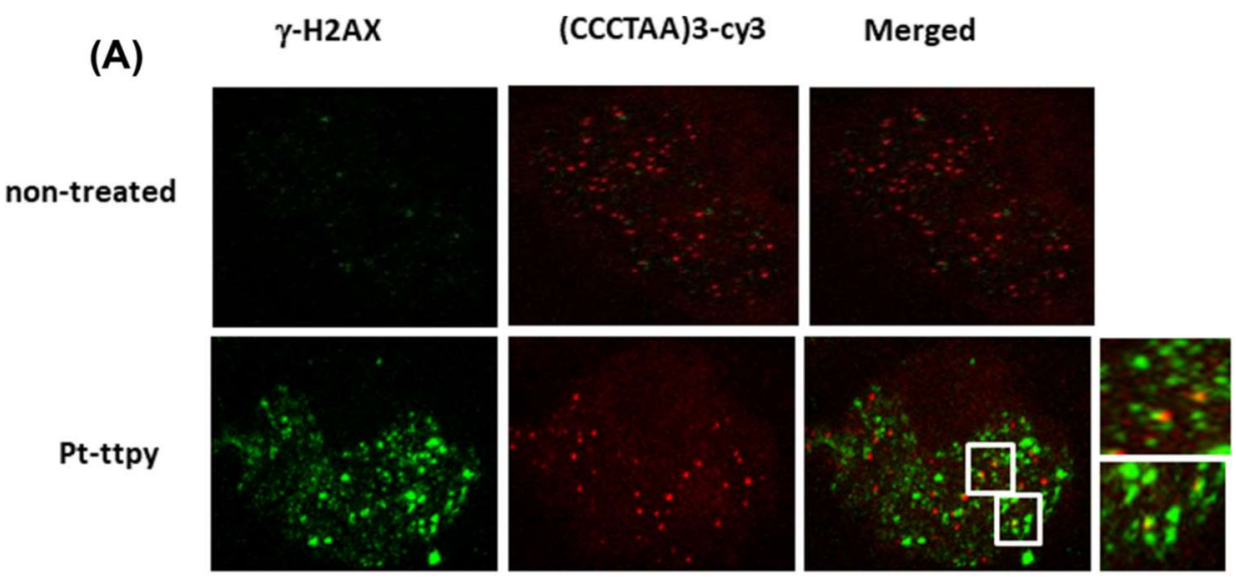

(B) $\square$ non-treated $\square$ cis-Pt $\square$ Pt-ttpy $\square$ Pt-tpy

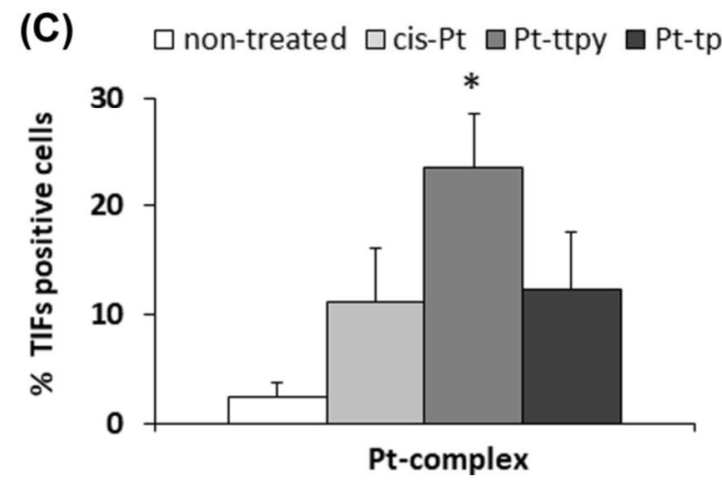

Fig. 3 DNA damage activation at telomeres. A2780 cells were treated for $96 \mathrm{~h}$ with Pt-ttpy, Pt-tpy, and cisplatin at their IC80 concentration. Cells were processed for immunofluorescence using antibodies against $\gamma-\mathrm{H} 2 \mathrm{AX}$ and C-rich PNA telomeric probe. (A) Z project of microscopy confocal acquisitions of cells treated with Pt-ttpy. (B) Percentages of $y$-H2AX positive cells. (C) Percentages of TIFs positive cells in untreated and treated cells. Cells with more than $20 \boldsymbol{\gamma}$-H2AX foci and more than three TIFs were scored as $\boldsymbol{\gamma}$-H2AX and TIF positive, respectively. (Mean of at least three experiments) Indicates a Mann and Withney test $P$-value $<0.05$ (GraphPad PRISM software

\section{TRF2 displacement from telomeres is induced upon treatment with Pt-ttpy and cisplatin but not with}

\section{Pt-tpy.}

Telomeric DNA damage, as assessed by TIFs quantification may be the consequence of telomere uncapping, especially by the removal from telomeres of TRF2 and POT1, two shelterin's proteins ensuring telomere protection. Indeed, some G4 ligands, including platinum complexes, were shown to dissociate TRF2 and/or POT1 from telomeres, leading to telomere uncapping and degradation $[41,58$ 64], and thus may result in telomere-end fusions [64-66]. Therefore, we investigated and compared the capacity of Pt-ttpy, Pt-tpy and cisplatin to induce telomere deprotection by quantifying the amount of TRF2, TRF1 and POT1 bound to telomeres after a 96h-treatment at an $\mathrm{IC}_{80}$ dose. Histone H3, a nontelomere specific DNA binding protein, was used as control.

Immunostaining of TRF2 first revealed that Pt-ttpy and cisplatin induced a significant loss of TRF2 foci (47 to 78\% remaining foci) in both A2780 (Figures 4A-B) and A2780cis cells (Figure 4C), whereas Pt-tpy had no impact. Since TRF2 foci are not exclusively localized at telomeres [67], we 
performed Chromatin Immunoprecipitation (ChIP) experiments using telomeric probes for the detection and quantification of DNA, which confirmed the immunostaining results. Indeed, 50 to $58 \%$ of TRF2 remained bound to telomeres in A2780 cells treated with cisplatin or Pt-ttpy, respectively, whereas TRF2 remained fully bound upon Pt-tpy treatment (Figure 5A-D). Similarly, in A2780cis cells, 56 to $68 \%$ of TRF2 remained bound to telomeres by cisplatin and Pt-ttpy treatments, respectively, whereas for Pt-tpy treatment, TRF2 remained fully bound (Figure 5E). In contrast, TRF1, POT1 and H3 were not affected and remained fully bound, irrespective of the platinum complex used.

Since the release of TRF2 from telomeres can also result from a decrease in TRF2 expression due to the stabilisation of the G4 structure located within its 5'UTR mRNA region [68], the amount of protein TRF2 was analysed by Western blot. TRF2 expression was not significantly affected by Pt-ttpy, Pt-tpy or cisplatin treatments (Figures 6A-B).

Since the release of TRF2 from telomeres can also result from its phosphorylation in position 188 [45], we checked pTRF2 formation by Western blot (Figure 6C). No TRF2 appeared phosphorylated on position 188 after $8 \mathrm{~h}$ treatment at 50mM Pt-tpy and Pt-ttpy. In contrast, only cisplatin clearly

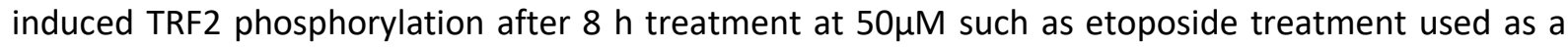
positive control.

Therefore, Pt-ttpy and cisplatin are able to induce specifically TRF2 removal from telomeres. This is expected for Pt-ttpy in a view of its ability to induce telomeric DNA damage and was already shown for cisplatin in HT1080 cell lines [64]. 

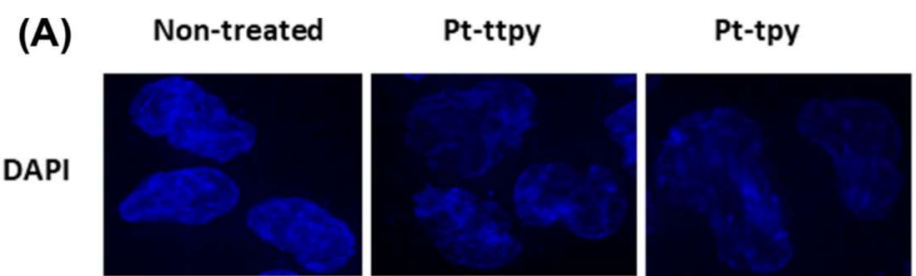

cis-Pt

TRF2
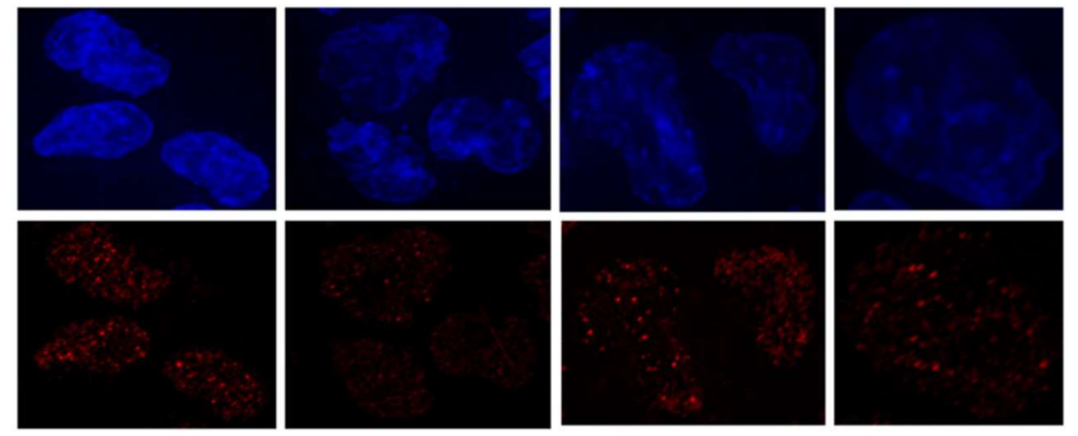

(B)

$\square$ cis-Pt $\quad \square$ Pt-ttpy $\square$ Pt-tpy $\square$ non-treated

(C) $\square$ cis-Pt $\square$ Pt-ttpy $\square$ Pt-tpy $\square$ non-treated
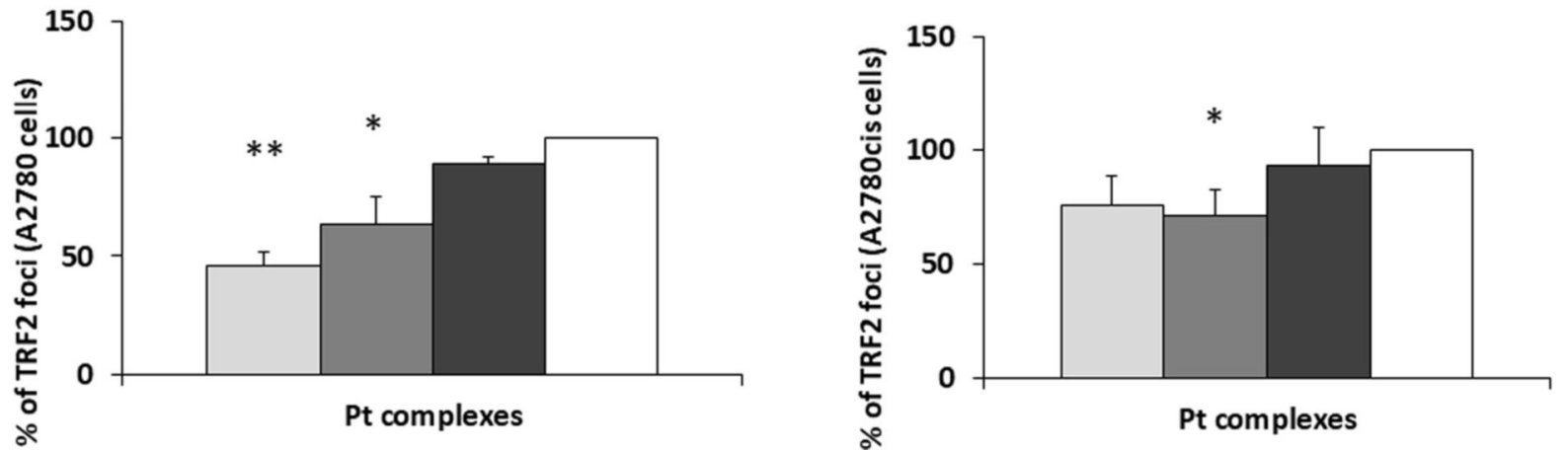

Fig. 4 TRF2 foci quantification detected by immunofluorescence on A2780 and A2780cis cells treated with cisplatin, Pt-ttpy, and Pt-tpy for $96 \mathrm{~h}$ at their respective IC80 concentration. (A) A2780 cells were processed for immunofluorescence using antibodies against TRF2. (B) \% of TRF2 foci after A2780 cell treatments with cisplatin, Pt-ttpy, and Pt-tpy. (C) \% of TRF2 foci after A2780cis cell treatments with cisplatin, Pt-ttpy, and Pt-tpy (mean of at least three experiments). ${ }^{* *}$ Indicates a Mann and Withney test $P$-value $<0.01$ and $* P<0.05$ (GraphPad PRISM software).

\section{Absence of telomere shortening upon TRF2 displacement from telomeres}

Since the release of TRF2 from telomeres can induce telomere shortening, we investigated the platinum complexes effects on telomere length by Southern blot and qPCR of telomeric DNA in A2780 cells. Southern blot analysis of the mean value of TRF (Telomere Restriction Fragments) and qPCR showed that Pt-ttpy, Pt-tpy, and cisplatin did not induce any telomere shortening in our conditions (Figures 6D and 6E). 
(A)

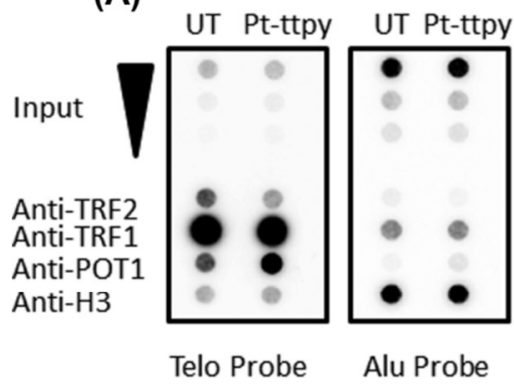

(B)

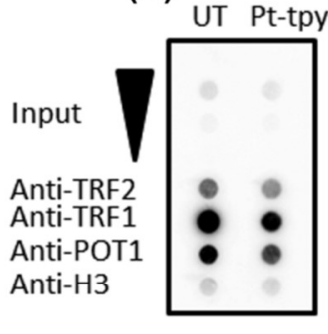

Telo Probe

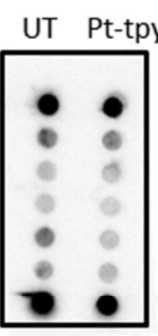

Alu Probe
(C)

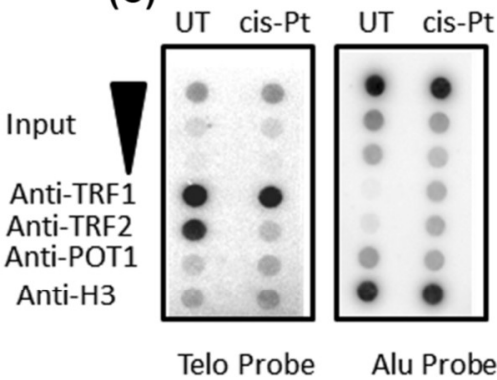

(D) $\square$ cis-pt $\quad \square$ Pt-ttpy $\quad \square$ Pt-tpy

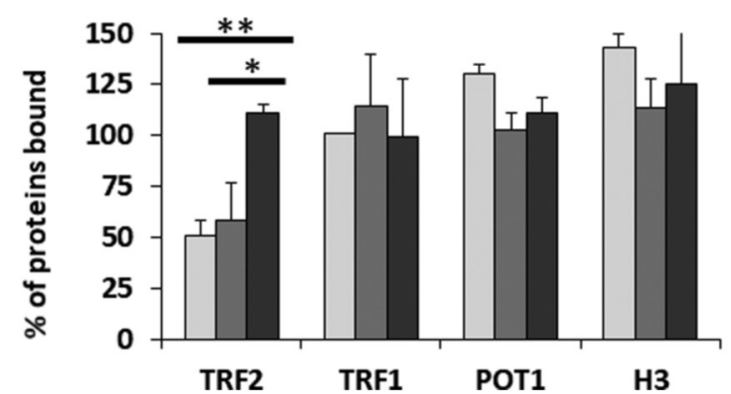

(E)

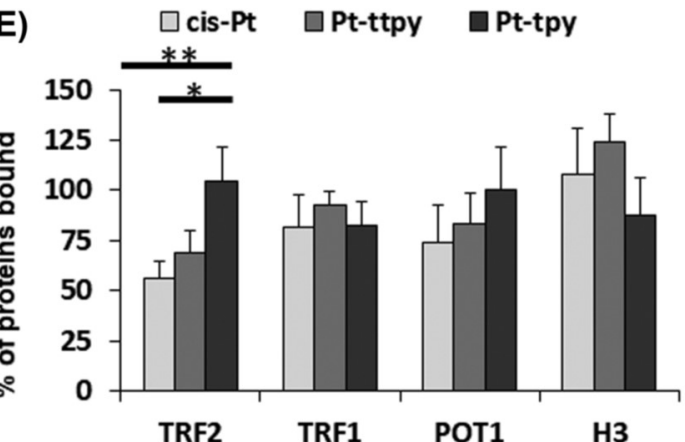

Fig. 5 Proteins bound to telomeres quantified from dot blots. Dot blot membranes of ChIP of TRF2, TRF1, POT1, and H3 on A2780 cells treated by doses of Pt-ttpy (A), Pt-tpy (B), and cisplatin (C) for $96 \mathrm{~h}$ at their respective IC80 concentration. (D) \% of proteins bound to telomeres of A2780 cells. (E) \% of proteins bound to telomeres of A2780cis cells. Telomeric sequences were evidenced in a DNA fraction immunoprecipitated by an anti-TRF1, anti-TRF2, anti-H3 antibody using a 32P radio labelled $800 \mathrm{pb}$ telomeric probe and normalized with $\alpha 32 \mathrm{P}$ radio labelled Alu sequences in untreated, cisplatin, Pt-ttpy, and Pt-tpy treated cells. $200 \mathrm{ng}$ of DNA were blotted for each sample. The \% represents the quantitative values of telomeric DNA signals in the samples originating from cells with treatment compared to the cells without any treatment. Quantitative values of telomeric DNA signals are calculated as the ratio between telomeric DNA signal precipitation and telomeric DNA signals in the INPUT for the same amount of blotted DNA. These values have been normalized by the amount of blotted DNA for each sample quantified by the non-specific Alu probe, following the formula: (telomere IP/telomere INPUT)/(Alu IP/Alu INPUT). (Means of at least three experiments) ** Indicates a Mann and Withney test $P$-value $<0.01$ and * $P<0.05$ (GraphPad PRISM software). Statistical analysis was made by comparing the amount of protein bound to telomeres for each treatment. 


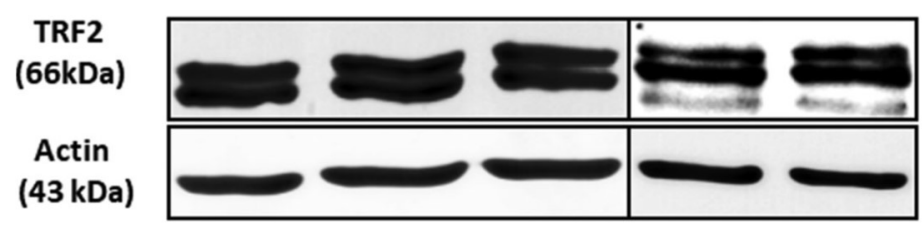

(B)
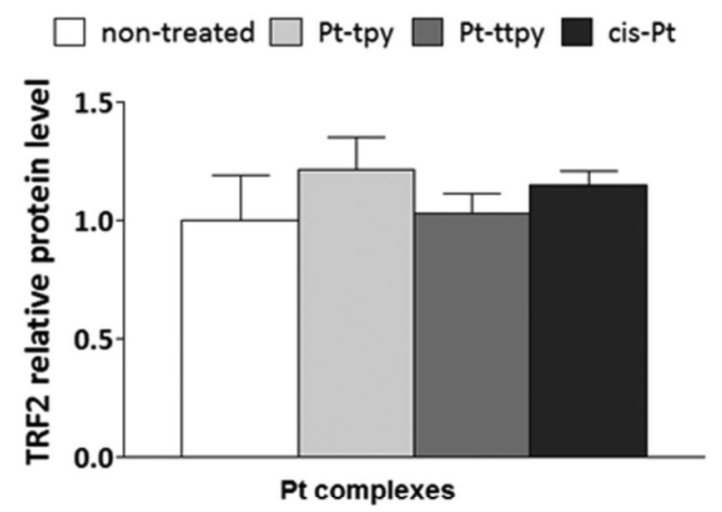

(C)

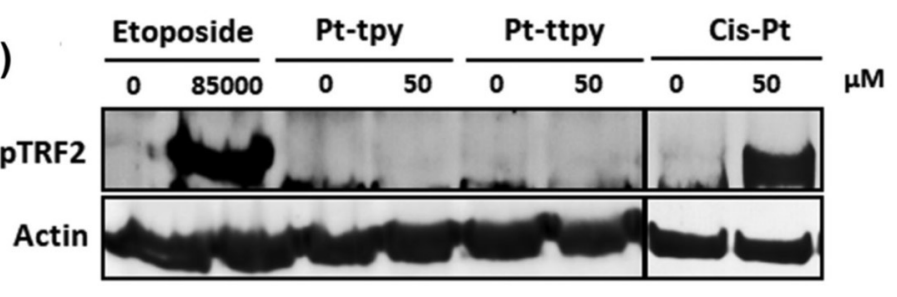

(E)
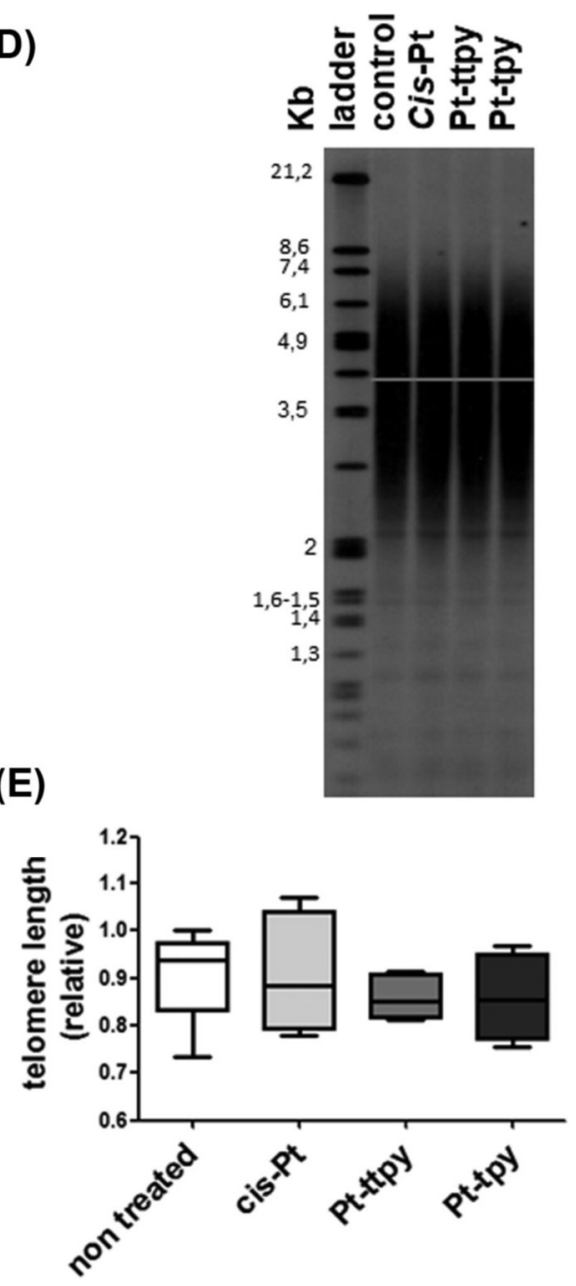

Fig. 6 Western blot of TRF2, pTRF2, and telomere length from A2780 treated cells with cisplatin, Pt-ttpy, and Pt-tpy for 96 h. (A) Western blot of TRF2 membranes, (B) TRF2 relative protein level normalized with actin (Mean of three experiments), (C) Western blot of pTRF2 membranes for $50 \mu \mathrm{M} 8 \mathrm{~h}$ treatment, and $85 \mathrm{mM}$ etoposide as positive control (D) Southern blot or (E) Relative telomere length measured by qPCR. The values were normalized using 36B4u gene (mean of three experiments).

\section{Pt-ttpy preferentially induced damage at genomic G-rich sequences but not exclusively at predicted G4 motifs}

$\gamma-\mathrm{H} 2 \mathrm{AX}$ foci induced upon drug treatment localize at telomeres but also in other chromosomal regions (Figures 3 and S3). In order to determine these sites, we conducted chromatin immunoprecipitation of cells treated with Pt-ttpy using an antibody against $\gamma-\mathrm{H} 2 \mathrm{AX}$ followed by NextGeneration sequencing (ChIP-seq), the same analysis was done in cisplatin-treated cells for the purpose of comparison and in non-treated cells used as control. The A2780 cells were treated with the $I_{80}$ doses of Pt-ttpy and cisplatin for 96 hours because under these conditions the two complexes reach their maximum of $40-50 \%$ of damage to genomic DNA.

We identified a total of $25,788,17,682$ and 10,050 peaks from treatments with cisplatin, Pt-ttpy and untreated cells, respectively (Figure 7A). Cisplatin and Pt-ttpy IPs were then analyzed over the 
untreated IPs, revealing 11744 common peaks (Figure 7A). Interestingly, the $\gamma-\mathrm{H} 2 \mathrm{AX}$ domains are unevenly distributed among chromosomes (Figure 7B). Indeed, $\gamma$-H2AX preferentially accumulates on six chromosomes, with the highest enrichment detected in chr1>chr21>chr16>chr15>chr9>chr19 (Figure 7C). Notably, this distribution is identical for cisplatin and Pt-ttpy and shows no link between the abundance of $\gamma-\mathrm{H} 2 \mathrm{AX}$ per chromosome with the length of the chromosomes, otherwise we would have the largest chromosomes (chr1, chr2, chr3, chrX, ...) amongst the most enriched. Of note, the bioinformatics analyses of the ChIP-seq data revealed also multiple reads that mapped onto mitochondrial DNA (chrM). Mitochondrial DNA exists in a closed-circular double-stranded form in high copy numbers in mitochondria within the cells as well as remnants of partial or whole copies within the nuclear genome, known as Nuclear Mitochondrial sequences (NUMTs) [69]. The ChIP-seq peaks that mapped on chrM covered almost the entire mitochondrial DNA. Thus, it was not possible to decipher whether the enriched signal originates from NUMTs or from mitochondria. Moreover, even if some histones like $\mathrm{H} 2 \mathrm{~A}$ and $\mathrm{H} 2 \mathrm{AX}$ were reported in mitochondria and that the decreased expression of $\mathrm{H} 2 \mathrm{AX}$ could lead to mitochondrial toxicity [70], no detectable $\gamma-\mathrm{H} 2 \mathrm{AX}$ has been reported within mitochondria, even under ionizing radiation [71]. Likewise, our immunofluorescence analyses did not show any $\gamma-\mathrm{H} 2 \mathrm{AX}$ signal outside from the nucleus upon drug treatments (Figures $3 \mathrm{~A}$ and S3A). In addition, we attempted to isolate mitochondria from cells in the presence of different treatments to confirm by WB that the DNA damage by $\gamma$-H2AX IF staining solely occurs in the nucleus. Unfortunately, the fraction of purified mitochondria was slightly contaminated by the presence of actin impeding the use the latter assay. These data suggest that chrM chipped by $\gamma-\mathrm{H} 2 \mathrm{AX}$ originates from the nucleus as NUMTs and not from mitochondria.

We observed that the preferred DNA damage domains are G- and A-rich tandemly repeated sequences, comprising satellite DNA, rDNA and pseudogene regions (Figure 8A). We then analyzed the enrichment of DNA damage sites in regions containing potential G4-forming sequences (G4L1-12 and G4L1-7 motifs, matching the regular expressions $\mathrm{G}_{3-5} \mathrm{~N}_{1-7} \mathrm{G}_{3-5} \mathrm{~N}_{1-7} \mathrm{G}_{3-5} \mathrm{~N}_{1-7} \mathrm{G}_{3-5}$ or $\mathrm{G}_{3-5} \mathrm{~N}_{1-12} \mathrm{G}_{3-5} \mathrm{~N}_{1-12} \mathrm{G}_{3-5} \mathrm{~N}_{1-}$ ${ }_{12} \mathrm{G}_{3-5}$; see Material and Methods) in gene promoters, telomeric sequences ([TTAGGG $]_{n}$ repeats) and in GG and GNG sites that are preferentially crosslinked by cisplatin [39] (Figure 8B). Except for GNGcontaining sequences, none of these domains, including G4 genomic motifs, are prominent damage domains of Pt-ttpy. Even if telomeric damages have been evidenced by confocal microscopy, we did not detect any telomeric enrichment in peaks from our sequencing data. However, since assigning telomeric reads with high confidence using ChIP-seq data is not possible (specialized software designed for WGS data, repetitive nature of the telomeric regions, long stretches of unknown nucleotides at the ends of most chromosomes in the human reference genome assembly), this quantification has not been considered in our analysis. For cisplatin, enrichment in $\gamma-\mathrm{H} 2 \mathrm{AX}$ domains containing GG and GNG was observed. To note, our genome wide pattern of $\gamma-\mathrm{H} 2 \mathrm{AX}$ domains of cisplatin treatments differs 
significantly from the maps obtained from cisplatin-DNA adducts and cisplatin DNA damage and repair sequencing of the human genome at single-nucleotide resolution $[72,73]$. Indeed, in our study, the $\gamma$ $\mathrm{H} 2 \mathrm{AX}$ domains do not correlate with the density of GG (Figure S4) and no enrichment has been observed in promoter regions. Most interestingly, the analysis of prominent peak regions highlights that six consensus motif sequences (60\% of the peaks) were significantly enriched after Pt-ttpy treatment and are different from those observed after cisplatin treatment (Figure S5).

Therefore, Pt-ttpy induced preferentially DNA damage in G- and A-rich tandemly repeated sequences in A2780 cells comprising satellite DNA, rDNA and pseudogene regions, and was not restricted to sites with G4-forming potential.

(A)
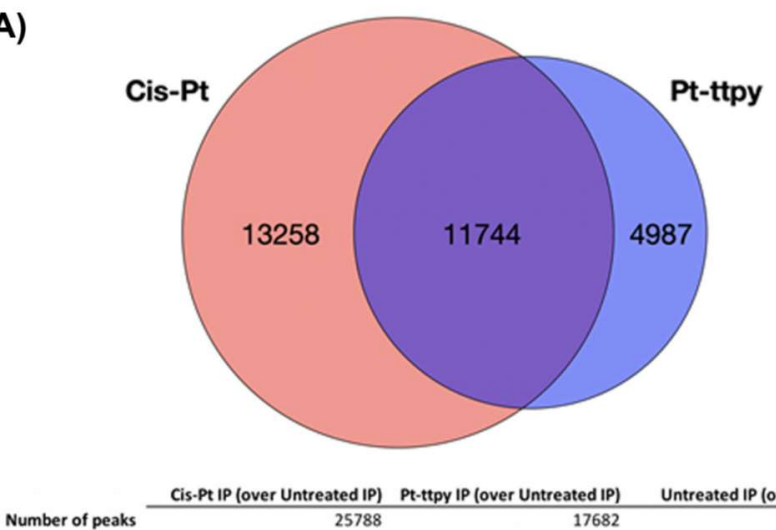$$
\text { - }
$$

(B)

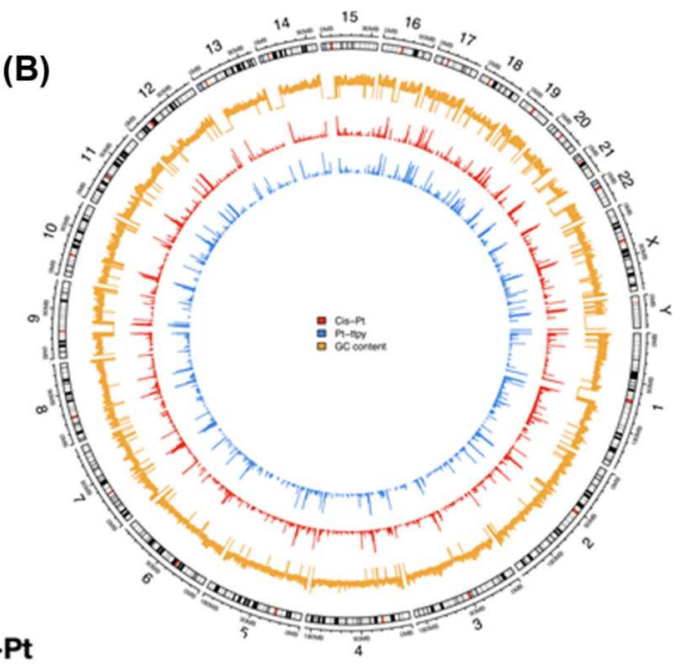

(C)

Pt-ttpy

Cis-Pt

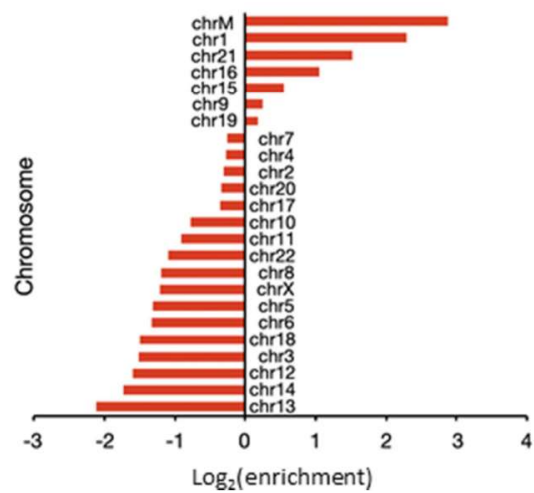

Fig. $7 \gamma-\mathrm{H} 2 \mathrm{AX}$ domains of cisplatin and Pt-ttpy are enriched in the same chromosomes, notably in mitochondrial DNA. (A) $\gamma$ -H2AX binding sites (peaks) detected in A2780 cells treated with cisplatin or Pt-ttpy. (B) Circular plot showing the genomewide peak distribution of cisplatin and Pt-ttpy $\boldsymbol{\gamma}$-H2AX IPs over the untreated $\boldsymbol{\gamma}$-H2AX IP. From the outermost to the innermost circle: orange, GC-content over all chromosomes; red, cisplatin $\boldsymbol{\gamma}$-H2AX peaks and; blue, Pt-ttpy $\boldsymbol{\gamma}$-H2AX peaks. (C) Relative peak enrichment on each chromosome and mtDNA annotated chrM. Blue, Pt-ttpy $\gamma-\mathrm{H} 2 \mathrm{AX}$ peaks; red, cisplatin $\gamma-\mathrm{H} 2 \mathrm{AX}$ peaks. 


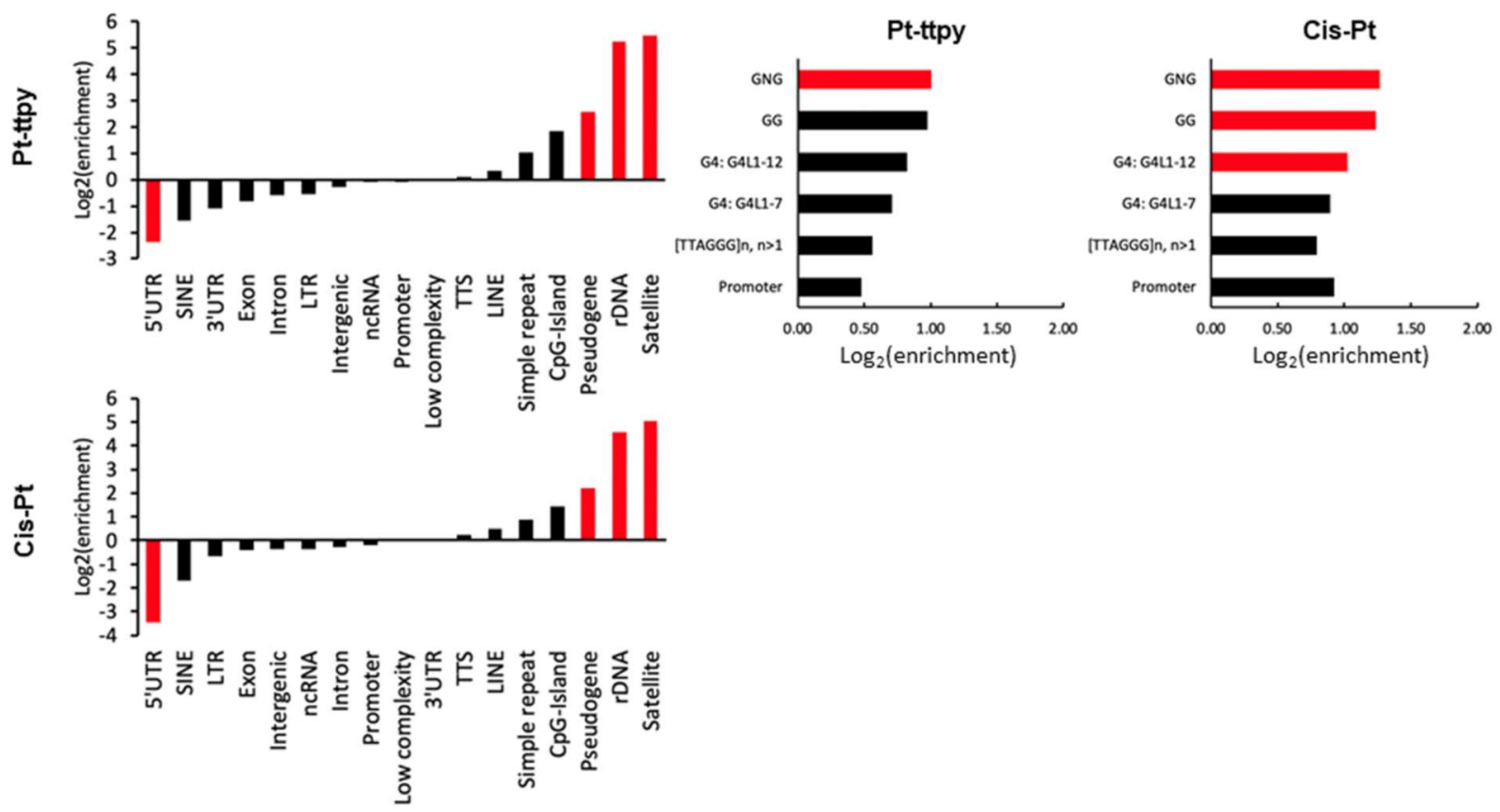

Fig. $8 \gamma-\mathrm{H} 2 \mathrm{AX}$ domains of cisplatin and Pt-ttpy are enriched in specific G- and A-rich sequences. (A) Annotation of cisplatin and Pt-ttpy $\gamma$-H2AX IPs over the untreated $\gamma$-H2AX IP. Top panel, Pt-ttpy treated cells; bottom panel, cisplatin treated cells. (B). Feature enrichments for cisplatin and Pt-ttpy $\gamma$-H2AX IPs over the untreated $\boldsymbol{\gamma}$-H2AX IP. Six G-rich motifs were assessed: telomeric repeats, [TTAGGG]n > 1; canonical G-quadruplex motifs with up to 7 (G4L1-7) or 12 nucleotides (G4L1-12) in the loops, G3-5N1-7G3-5N1-7G3-5N1-7G3-5 or G3-5N1-12G3-5N1-12G3-5N1-12G3-5; GG, GA or GNG sites; as well as the promoter annotation feature 


\section{Discussion}

Here, we examined the cellular and molecular targets of the G4 ligand tolylterpyridine platinum complex, Pt-ttpy $[33,34]$, that can bind and trap G4s irreversibly by metallic mono-coordination [30, 35]. We used, for comparison, the structurally related compound Pt-tpy that displays weak G4-binding, and another prevalent anti-cancer drug, cisplatin, that mainly binds DNA by bis-coordination between two adjacent guanines. Due to the dual properties of Pt-ttpy (G4 ligand and platinum coordinating complex), we analyzed its ability to induce telomere damage and identified other genomic DNA damage sites.

In the context of the search for platinum complexes able to overcome cisplatin resistance [74], Pt-ttpy and Pt-tpy are promising complexes from a pharmacological viewpoint, since they do not exhibit crossresistance with cisplatin [34]. Indeed, both complexes overcome the reduced influx and enhanced efflux of cisplatin contributing to cisplatin resistance in the A2780cis cell line, in comparison to its sensitive A2780 counterpart (Table 1, Figure 2) [53]. Interestingly, in A2780 cells, while the cellular uptake of both Pt-ttpy and Pt-tpy is greatly increased (7 to 16-fold) in comparison with cisplatin at isoeffect doses, the amount of platinum bound to DNA remains in the same order of magnitude (factor 0.7 to 1.6$)$ for all complexes. This suggests that their anti-proliferative activity is mainly related to their DNA binding activity, as previously proposed for many platinum complexes [39, 75]. Conversely, in A2780cis cells, the amount of platinum bound to DNA after cisplatin, Pt-ttpy and Pt-tpy treatments is not at the same level (Table 1). This indicates that their capacity to promote Pt-DNA adducts cannot entirely explain the drugs anti-cancer activities in A2780cis cells, that are supposed to have a high tolerance to DNA damage [76]. This also indicates that circumventing cisplatin resistance involves many molecular and cellular targets that need to be characterized [77]. Therefore, to get further insight into the mechanism of Pt-ttpy anti-cancer activities, it was important to decipher whether the effects of Pt-ttpy were dependent on its preferential DNA structure recognition (G4 versus duplex DNA).

Firstly, the telomere target of Pt-ttpy was uncovered: Pt-ttpy induced telomeric DNA damage concomitantly with a partial release of TRF2 from telomeres. Interestingly, telomeric DNA damage by Pt-ttpy treatment has already been highlighted using TRF2 as a telomeric probe [38], indicating that the remaining bound TRF2 still localizes with telomeric DNA damage.The telomere targeting of Pt-ttpy also agrees with previous finding showing that Pt-ttpy is able to induce efficiently and exclusively chromosome loss in linear (containing telomeres) but not in circular (lacking telomeres) human chromosome [38]. As found for Pt-ttpy, G4 ligands may induce partial uncapping TRF2 and /or POT1 from telomeres and telomeric damages $[41,58-64,78-80]$. Our results can provide some insights 
concerning the mechanism of TRF2 delocalization from telomeres that not exclusively the property of G4 ligands, since cisplatin succeeded, and telomeric DNA damage induction by drug cell treatments. The release of TRF2 from telomeres may originate from various processes. It could be due to a sterically impediment as shown in vitro on telomeric DNA fragments platinated by cisplatin [81], or with the G4 ligand telomestatin $[82,83]$. It could also originate from the phosphorylation status of TRF2 since its phosphorylation in position 188 reduces TRF2's affinity for telomeres [45]. TRF2 phosphorylation occurs rapidly in response to DNA damage such as IR or etoposide [45] [84]. We indeed show that cisplatin for higher concentration treatment and short time treatment, but not Pt-ttpy, induces the phosphorylation of TRF2 in position 188 which may consequently contribute to TRF2 release from telomeres in these conditions [37] . Moreover, since TRF2 is involved in many extratelomeric functions, and in particular in the response to DNA damage [85], it should be also interesting to evaluate the extratelomeric target of TRF2 released from telomeres upon the various treatments. Concerning telomeric DNA damage, since the shelterin complex at telomeres is essential to cell viability, in particular its TRF2 and POT1 components that block the activation of DNA damage responses by ATM and ATR respectively at telomeres $[13,86]$, it has been proposed that the removal of TRF2/POT1 from telomeres could consequently lead to telomeric DNA damage, telomere dysfunction and consequently participate to cell death $[13,87]$. However this is not the case of cisplatin thereby confirming previous findings [64]. Consequently, this suggests that there is no correlation between telomeric DNA damage and the partial removal of TRF2. Telomeric damage could be better induced by replication and transcription impairments due to G4 stabilization that are well known to lead to DNA double strand breaks [88]. Therefore, our results suggest that telomeric effect of Pt-ttpy is more connected to its G4 binding capacity rather than to its platination properties.

Secondly, at genome-wide level, $\gamma-\mathrm{H} 2 \mathrm{AX}$ chromatin immunoprecipitation followed by NextGeneration-Sequencing (ChIP-seq) showed that the $\gamma-\mathrm{H} 2 \mathrm{AX}$ domains of Pt-ttpy treatment did not accumulate in potential G4-forming sequences (PQS) irrespective of the search criterion used (G4L112 and G4L1-7) [3, 89] and 2) but followed the same enrichment pattern as cisplatin. This strongly suggests that the preferential genomic DNA sequence of Pt-ttpy-induced damage response through $\gamma$ $\mathrm{H} 2 \mathrm{AX}$ is not driven by G4-binding properties, but rather its platination properties, in contrast to the $\mathrm{G} 4$ ligand Pyridostatin for which $\gamma-\mathrm{H} 2 \mathrm{AX}$ domains have been mainly firstly found in oncogene promoters containing PQS [90] but subsequently shown to occur at other loci [91]. In addition, six prominent consensus DNA damaged sequences were defined to be unique to the Pt-ttpy treatment as compared to cisplatin. This could be due to a differential ability of the platinum complexes to form various DNAadducts, as mono adducts for Pt-ttpy and intra- and inter-strand crosslinks for cisplatin, that will be processed differently during replication, transcription and/or repair leading consequently to various 
DNA damage sites [92]. This singular property may be an important feature for the anti-cancer activity of Pt-ttpy.

It should be noted that the genome-wide pattern of cisplatin $\gamma-\mathrm{H} 2 \mathrm{AX}$ domains obtained herein, that is enriched in G- and A-rich sequences, differs significantly from the single nucleotide resolution maps of cisplatin-DNA adducts and cisplatin repair sites recently reported [72, 73, 92]. In these studies, the cisplatin-DNA adduct distribution (using antibodies against cisplatin and high mobility group protein HMGB1 for IP) appeared dictated primarily by the GG frequency whereas the cisplatin repair sites (using antibodies against TFIIH for IP) were shown to be highly heterogeneous and significantly correlated to transcription and chromatin states. To explain this discordance, it was proposed that the chromatin state would limit the accessibility of the Nucleotide Excision Repair (NER machinery), thus impairing its activity $[93,94]$. Consequently, the persistence of DBS and of $\gamma-\mathrm{H} 2 \mathrm{AX}$ domains spreading over many kb would depend on the repair efficiency. These arguments could also explain the $\gamma-\mathrm{H} 2 \mathrm{AX}$ domains patterns reported herein. In fact, although the detection of $\gamma-\mathrm{H} 2 \mathrm{AX}$ has been used to identify G4-drug binding sites on DNA [90], our findings indicate that this approach may reflect only partly the DNA-drug binding sites, at least in the context of drugs with DNA metal coordination capacity.

In conclusion, we showed that Pt-ttpy presents unique features in terms of cellular targets as compared to cisplatin. Pt-ttpy targets telomeres and induces their dysfunction, probably by G4 recognition, while its main genomic DNA damage sequences are not related to its G4 recognition properties. Hence, the in cellulo activities of Pt-ttpy can be exploited in order to circumvent increasing cisplatin resistance in chemotherapy. 


\section{Author contribution:}

Conceptualization, S.B; Conceptualization and Investigation bioinformatics, E.P-L, A.L-V, A.N; methodology, investigation, S.A, D.G, T.J, G.V, L.A, J.P, S.B; writing-original draft preparation, S.B, E.PL; writing-review and editing, S.B, E.P-L, D.G, T.J, M-P.T-F, A.L-V, A.N; supervision, SB, A.L-V, A.N.

\section{Acknowledgments:}

We thank David Gilley and Nazmul Huda for providing Anti-TRF2 Thr-188P polyclonal antibody. We sincerely acknowledge the SCM (Service Commun de Microscopie -Faculté des Sciences Fondamentales et Biomedicales - Université Paris Descartes) and Charlène Lasgi for technical help on flow cytometry (Flow cytometry Orsay facility, Curie). High-throughput sequencing was performed by the ICGex NGS platform of the Institut Curie supported by the grants ANR-10-EQPX-03 (Equipex) and ANR-10-INBS-09-08 (France Génomique Consortium) from the Agence Nationale de la Recherche ("Investissements d'Avenir" program), by the ITMO-Cancer Aviesan (Plan Cancer III) and by the SiRICCurie program (SiRIC Grant INCa-DGOS- 465 and INCa-DGOS-Inserm_12554).

\section{Founding}

This research was funded by CNRS, INSERM, Institut Curie, Paris-Saclay University, Association pour la Recherche contre le Cancer (ARC grant for DP), the Institut National du Cancer INCA, (2010-1-PLBIO)04UP5-14835 grant). This project has received funding from the European Union's Horizon 2020 research and innovation program under the Marie Skłodowska-Curie grant agreement No 666003 (Grant for DP) and Institut Curie (Grant for TJ).

\section{Conflict of Interest}

The authors declare no questions of bias and conflicts of interest in this work.

\section{Data availability}

ChIP-seq data were deposited into the Gene Expression Omnibus (GEO) database, under accession number GSE171450. The data used to support the findings of this study are available from the corresponding author upon request 


\section{References}

1. Burge, S., Parkinson, G. N., Hazel, P., Todd, A. K. \& Neidle, S. (2006) Quadruplex DNA: sequence, topology and structure, Nucleic Acids Res. 34, 5402-15.

2. Huppert, J. L. \& Balasubramanian, S. (2007) G-quadruplexes in promoters throughout the human genome, Nucleic Acids Res. 35, 406-13.

3. Bedrat, A., Lacroix, L. \& Mergny, J. L. (2016) Re-evaluation of G-quadruplex propensity with G4Hunter, Nucleic Acids Res. 44, 1746-59.

4. Puig Lombardi, E. \& Londoño-Vallejo, A. (2019) A guide to computational methods for Gquadruplex prediction, Nucleic Acids Res. 48, 1-15.

5. Chambers, V. S., Marsico, G., Boutell, J. M., Di Antonio, M., Smith, G. P. \& Balasubramanian, S. (2015) High-throughput sequencing of DNA G-quadruplex structures in the human genome, Nat Biotechnol.

6. Hansel-Hertsch, R., Beraldi, D., Lensing, S. V., Marsico, G., Zyner, K., Parry, A., Di Antonio, M., Pike, J., Kimura, H., Narita, M., Tannahill, D. \& Balasubramanian, S. (2016) G-quadruplex structures mark human regulatory chromatin, Nat Genet. 48, 1267-72.

7. Spiegel, J., Adhikari, S. \& Balasubramanian, S. (2020) The Structure and Function of DNA GQuadruplexes, Trends in Chemistry. 2, 123-136.

8. Varshney, D., Spiegel, J., Zyner, K., Tannahill, D. \& Balasubramanian, S. (2020) The regulation and functions of DNA and RNA G-quadruplexes, Nature Reviews Molecular Cell Biology. 21, 459-474.

9. Biffi, G., Tannahill, D., McCafferty, J. \& Balasubramanian, S. (2013) Quantitative visualization of DNA G-quadruplex structures in human cells, Nat Chem. 5, 182-6.

10. Granotier, C., Pennarun, G., Riou, L., Hoffschir, F., Gauthier, L. R., De Cian, A., Gomez, D., Mandine, E., Riou, J. F., Mergny, J. L., Mailliet, P., Dutrillaux, B. \& Boussin, F. D. (2005) Preferential binding of a G-quadruplex ligand to human chromosome ends, Nucleic Acids Res. 33, 4182-90.

11. Blasco, M. A. (2005) Telomeres and human disease: ageing, cancer and beyond, Nat Rev Genet. 6, 611-22.

12. Palm, W. \& de Lange, T. (2008) How shelterin protects mammalian telomeres, Annu Rev Genet. 42, 301-34.

13. de Lange, T. (2018) Shelterin-Mediated Telomere Protection, Annu Rev Genet. 52, 223-247.

14. Ivancich, M., Schrank, Z., Wojdyla, L., Leviskas, B., Kuckovic, A., Sanjali, A. \& Puri, N. (2017) Treating Cancer by Targeting Telomeres and Telomerase, Antioxidants. 6.

15. Rhodes, D. \& Lipps, H. J. (2015) G-quadruplexes and their regulatory roles in biology, Nucleic Acids Res. 43, 8627-37.

16. Maizels, N. (2012) G4 motifs in human genes, Ann N Y Acad Sci. 1267, 53-60. 
17. Murat, P. \& Balasubramanian, S. (2014) Existence and consequences of G-quadruplex structures in DNA, Curr Opin Genet Dev. 25, 22-29.

18. Gray, L. T., Vallur, A. C., Eddy, J. \& Maizels, N. (2014) G quadruplexes are genomewide targets of transcriptional helicases XPB and XPD, Nat Chem Biol. 10, 313-8.

19. Bugaut, A. \& Balasubramanian, S. (2012) 5'-UTR RNA G-quadruplexes: translation regulation and targeting, Nucleic Acids Res. 40, 4727-41.

20. Monchaud, D. \& Teulade-Fichou, M. P. (2008) A hitchhiker's guide to G-quadruplex ligands, Org Biomol Chem. 6, 627-36.

21. Asamitsu, S., Obata, S., Yu, Z., Bando, T. \& Sugiyama, H. (2019) Recent Progress of Targeted GQuadruplex-Preferred Ligands Toward Cancer Therapy, Molecules. 24.

22. Georgiades, S. N., Karim, N. H. A., Kogularamanan Suntharalingam \& Vilar, R. (2010) Interaction of Metal Complexes with G-Quadruplex DNA, Angew Chem Int Ed. 49, 4020-4034.

23. Vilar, R. (2018) Nucleic Acid Quadruplexes and Metallo-Drugs, Metal ions in life sciences. 18, 325349.

24. Müller, S. \& Rodriguez, R. (2014) G-quadruplex interacting small molecules and drugs: from bench toward bedside, Expert Rev Clin Pharmacol. 7, 663-79.

25. Ahmed, A. A., Marchetti, C., Ohnmacht, S. A. \& Neidle, S. (2020) A G-quadruplex-binding compound shows potent activity in human gemcitabine-resistant pancreatic cancer cells, Scientific reports. 10, 12192.

26. Carvalho, J., Mergny, J. L., Salgado, G. F., Queiroz, J. A. \& Cruz, C. (2020) G-quadruplex, Friend or Foe: The Role of the G-quartet in Anticancer Strategies, Trends in molecular medicine. 26, 848-861. 27. Rao, L. \& Bierbach, U. (2007) Kinetically favored platination of adenine in the g-rich human telomeric repeat, J Am Chem Soc. 129, 15764-5.

28. Rao, L., Dworkin, J. D., Nell, W. E. \& Bierbach, U. (2011) Interactions of a Platinum-Modified Perylene Derivative with the Human Telomeric G-Quadruplex, J Phys Chem B. 115, 13701-13712. 29. Bertrand H, Bombard S, Monchaud D \& Teulade-Fichou, M. P. (2007) A Platinum-Quinacridine Hybrid as G-Quadruplex Ligand, J Biol Inorg Chem. 12, 1003-1014.

30. Bertrand, H., Bombard, S., Monchaud, D., Talbot, E., Guedin, A., Mergny, J. L., Grunert, R., Bednarski, P. J. \& Teulade-Fichou, M. P. (2009) Exclusive platination of loop adenines in the human telomeric G-quadruplex, Org Biomol Chem. 7, 2864-71.

31. Betzer, J. F., Nuter, F., Chtchigrovsky, M., Hamon, F., Kellermann, G., Ali, S., M.A., C., Roque, S., Poupon, J., Cresteil, T., Teulade-Fichou, M. P., Marinetti, A. \& Bombard, S. (2016) Linking of antitumour trans NHC-Pt(II) complexes to G-quadruplex DNA ligand for telomeric targeting, Bioconjug Chem. 27, 1456. 
32. He, L., Meng, Z., Xu, D. \& Shao, F. (2018) Dual functional dinuclear platinum complex with selective reactivity towards c-myc G-quadruplex, Scientific reports. 8, 767.

33. Bertrand, H., Monchaud, D., De Cian, A., Guillot, R., Mergny, J. L. \& Teulade-Fichou, M. P. (2007) The importance of metal geometry in the recognition of G-quadruplex-DNA by metal-terpyridine complexes, Org Biomol Chem. 5, 2555-9.

34. Morel, E., Beauvineau, C., Naud-Martin, D., Landras-Guetta, C., Verga, D., Ghosh, D., Achelle, S., Mahuteau-Betzer, F., Bombard, S. \& Teulade-Fichou, M. P. (2019) Selectivity of Terpyridine Platinum Anticancer Drugs for G-quadruplex DNA, Molecules. 24.

35. Trajkovski, M., Morel, E., Hamon, F., Bombard, S., Teulade-Fichou, M. P. \& Plavec, J. (2015) Interactions of Pt-ttpy with G-Quadruplexes Originating from Promoter Region of the c-myc Gene Deciphered by NMR and Gel Electrophoresis Analysis, Chem Eur J. 21, 7798-807.

36. Merle, P., Gueugneau, M., Teulade-Fichou, M. P., Müller-Barthélémy, M., Amiard, S., Chautard, E., Guetta, C., Dedieu, V., Communal, Y., Mergny, J. L., Gallego, M., White, C., Verrelle, P. \& Tchirkov, A. (2015) Highly efficient radiosensitization of human glioblastoma and lung cancer cells by a Gquadruplex DNA binding compound, Sci Rep. 5, 16255-16266.

37. Saker, L., Ali, S., Masserot, C., Kellermann, G., Poupon, J., Teulade-Fichou, M. P., SegalBendirdjian, E. \& Bombard, S. (2018) Platinum Complexes Can Bind to Telomeres by Coordination, International journal of molecular sciences. 19, 1951-1966.

38. Lee, H. S., Carmena, M., Liskovykh, M., Peat, E., Kim, J. H., Oshimura, M., Masumoto, H., TeuladeFichou, M. P., Pommier, Y., Earnshaw, W. C., Larionov, V. \& Kouprina, N. (2018) Systematic Analysis of Compounds Specifically Targeting Telomeres and Telomerase for Clinical Implications in Cancer Therapy, Cancer Res. 78, 6282-6296.

39. Jung, Y. \& Lippard, S. J. (2007) Direct cellular responses to platinum-induced DNA damage, Chem Rev. 107, 1387-407.

40. Chan, G. K., Kleinheinz, T. L., Peterson, D. \& Moffat, J. G. (2013) A simple high-content cell cycle assay reveals frequent discrepancies between cell number and ATP and MTS proliferation assays, PLoS One. 8, e63583.

41. Betzer, J. F., Nuter, F., Chtchigrovsky, M., Hamon, F., Kellermann, G., Ali, S., Calmejane, M. A., Roque, S., Poupon, J., Cresteil, T., Teulade-Fichou, M. P., Marinetti, A. \& Bombard, S. (2016) Linking of Antitumor trans NHC-Pt(II) Complexes to G-Quadruplex DNA Ligand for Telomeric Targeting, Bioconjug Chem. 27, 1456-1470.

42. Amiard, S., Doudeau, M., Pinte, S., Poulet, A., Lenain, C., Faivre-Moskalenko, C., Angelov, D., Hug, N., Vindigni, A., Bouvet, P., Paoletti, J., Gilson, E. \& Giraud-Panis, M. J. (2007) A topological mechanism for TRF2-enhanced strand invasion, Nat Struct Mol Biol. 14, 147-54.

43. Cawthon, R. M. (2002) Telomere measurement by quantitative PCR, Nucleic Acids Res. 30, e47. 
44. Gil, M. E. \& Coetzer, T. L. (2004) Real-time quantitative PCR of telomere length, Mol Biotechnol. 27, 169-72.

45. Tanaka, H., Mendonca, M. S., Bradshaw, P. S., Hoelz, D. J., Malkas, L. H., Meyn, M. S. \& Gilley, D. (2005) DNA damage-induced phosphorylation of the human telomere-associated protein TRF2, Proc Natl Acad Sci U S A. 102, 15539-44.

46. Li, H. (2013) Aligning sequence reads, clone sequences and assembly contigs with BWA-MEM, arXivorg 1303.3997v2.

47. Zhang, Y., Liu, T., Meyer, C. A., Eeckhoute, J., Johnson, D. S., Bernstein, B. E., Nusbaum, C., Myers, R. M., Brown, M., Li, W. \& Liu, X. S. (2008) Model-based analysis of ChIP-Seq (MACS), Genome Biol. 9, R137.

48. R Core Team R (2017) R: A language and environment for statistical computing. R Foundation for Statistical Computing,, Vienna, Austria URL https://wwwR-projectorg

49. Heinz, S., Benner, C., Spann, N., Bertolino, E., Lin, Y. C., Laslo, P., Cheng, J. X., Murre, C., Singh, H. \& Glass, C. K. (2010) Simple combinations of lineage-determining transcription factors prime cisregulatory elements required for macrophage and B cell identities, Mol Cell. 38, 576-89.

50. Chaney, S. G., Campbell, S. L., Bassett, E. \& Wu, Y. (2005) Recognition and processing of cisplatinand oxaliplatin-DNA adducts, Crit Rev Oncol Hematol. 53, 3-11.

51. Solomatina, A. I., Chelushkin, P. S., Krupenya, D. V., Podkorytov, I. S., Artamonova, T. O., Sizov, V. V., Melnikov, A. S., Gurzhiy, V. V., Koshel, E. I., Shcheslavskiy, V. I. \& Tunik, S. P. (2017) Coordination to Imidazole Ring Switches on Phosphorescence of Platinum Cyclometalated Complexes: The Route to Selective Labeling of Peptides and Proteins via Histidine Residues, Bioconjug Chem. 28, 426-437.

52. Corte Rodriguez, M., Alvarez-Fernandez Garcia, R., Blanco, E., Bettmer, J. \& Montes-Bayon, M. (2017) Quantitative Evaluation of Cisplatin Uptake in Sensitive and Resistant Individual Cells by Single-Cell ICP-MS (SC-ICP-MS), Anal Chem. 89, 11491-11497.

53. Amable, L. (2016) Cisplatin resistance and opportunities for precision medicine, Pharmacological research. 106, 27-36.

54. Safaei, R., Larson, B. J., Cheng, T. C., Gibson, M. A., Otani, S., Naerdemann, W. \& Howell, S. B. (2005) Abnormal lysosomal trafficking and enhanced exosomal export of cisplatin in drug-resistant human ovarian carcinoma cells, Mol Cancer Ther. 4, 1595-604.

55. Groessl, M., Zava, O. \& Dyson, P. J. (2011) Cellular uptake and subcellular distribution of ruthenium-based metallodrugs under clinical investigation versus cisplatin, Metallomics. 3, 591-9. 56. Lee, R. F. S., Riedel, T., Escrig, S., Maclachlan, C., Knott, G. W., Davey, C. A., Johnsson, K., Meibom, A. \& Dyson, P. J. (2017) Differences in cisplatin distribution in sensitive and resistant ovarian cancer cells: a TEM/NanoSIMS study, Metallomics. 9, 1413-1420. 
57. Takai, H., Smogorzewska, A. \& de Lange, T. (2003) DNA damage foci at dysfunctional telomeres, Curr Biol. 13, 1549-56.

58. Tahara, H., Shin-Ya, K., Seimiya, H., Yamada, H., Tsuruo, T. \& Ide, T. (2006) G-Quadruplex stabilization by telomestatin induces TRF2 protein dissociation from telomeres and anaphase bridge formation accompanied by loss of the 3 ' telomeric overhang in cancer cells, Oncogene. 25, 1955-66. 59. Gomez, D., O'Donohue, M. F., Wenner, T., Douarre, C., Macadre, J., Koebel, P., Giraud-Panis, M. J., Kaplan, H., Kolkes, A., Shin-ya, K. \& Riou, J. F. (2006) The G-quadruplex ligand telomestatin inhibits POT1 binding to telomeric sequences in vitro and induces GFP-POT1 dissociation from telomeres in human cells, Cancer Res. 66, 6908-12.

60. Zhou, W. J., Deng, R., Zhang, X. Y., Feng, G. K., Gu, L. Q. \& Zhu, X. F. (2009) G-quadruplex ligand SYUIQ-5 induces autophagy by telomere damage and TRF2 delocalization in cancer cells, Mol Cancer Ther. 8, 3203-13.

61. Pagano, B., Amato, J., laccarino, N., Cingolani, C., Zizza, P., Biroccio, A., Novellino, E. \& Randazzo, A. (2015) Looking for efficient G-quadruplex ligands: evidence for selective stabilizing properties and telomere damage by drug-like molecules, ChemMedChem. 10, 640-9.

62. Rizzo, A., lachettini, S., Zizza, P., Cingolani, C., Porru, M., Artuso, S., Stevens, M., Hummersone, M., Biroccio, A., Salvati, E. \& Leonetti, C. (2014) Identification of novel RHPS4-derivative ligands with improved toxicological profiles and telomere-targeting activities, Journal of experimental \& clinical cancer research : CR. 33, 81-89.

63. Qin, H., Zhao, C., Sun, Y., Ren, J. \& Qu, X. (2017) Metallo-supramolecular Complexes Enantioselectively Eradicate Cancer Stem Cells in Vivo, J Am Chem Soc. 139, 16201-16209. 64. Charif, R., Granotier-Beckers, C., Bertrand, H. C., Poupon, J., Ségal-Bendirdjian, E., TeuladeFichou, M. P., Boussin, F. D. \& Bombard, S. (2017) Association of a platinum complex to a Gquadruplex ligand enhances the telomere disruption, Chem ResTox. 38, 1629-1640.

65. Pennarun, G., Granotier, C., Gauthier, L. R., Gomez, D., Hoffschir, F., Mandine, E., Riou, J. F., Mergny, J. L., Mailliet, P. \& Boussin, F. D. (2005) Apoptosis related to telomere instability and cell cycle alterations in human glioma cells treated by new highly selective G-quadruplex ligands, Oncogene. 24, 2917-28.

66. Brassart, B., Gomez, D., De Cian, A., Paterski, R., Montagnac, A., Qui, K. H., Temime-Smaali, N., Trentesaux, C., Mergny, J. L., Gueritte, F. \& Riou, J. F. (2007) A new steroid derivative stabilizes gquadruplexes and induces telomere uncapping in human tumor cells, Mol Pharmacol. 72, 631-40. 67. Simonet, T., Zaragosi, L. E., Philippe, C., Lebrigand, K., Schouteden, C., Augereau, A., Bauwens, S., Ye, J., Santagostino, M., Giulotto, E., Magdinier, F., Horard, B., Barbry, P., Waldmann, R. \& Gilson, E. (2011) The human TTAGGG repeat factors 1 and 2 bind to a subset of interstitial telomeric sequences and satellite repeats, Cell Res. 21, 1028-38. 
68. Gomez, D., Guedin, A., Mergny, J. L., Salles, B., Riou, J. F., Teulade-Fichou, M. P. \& Calsou, P. (2010) A G-quadruplex structure within the 5'-UTR of TRF2 mRNA represses translation in human cells, Nucleic Acids Res.

69. Tsuji, J., Frith, M. C., Tomii, K. \& Horton, P. (2012) Mammalian NUMT insertion is non-random, Nucleic Acids Res. 40, 9073-88.

70. Jeong, J. H., Cheol Kang, Y., Piao, Y., Kang, S. \& Pak, Y. K. (2017) miR-24-mediated knockdown of $\mathrm{H} 2 \mathrm{AX}$ damages mitochondria and the insulin signaling pathway, Experimental \& molecular medicine. 49, e313.

71. Choi, Y. S., Jeong, J. H., Min, H. K., Jung, H. J., Hwang, D., Lee , S. W. \& Pak, Y. K. (2011) Shot-gun proteomic analysis of mitochondrial D-loop DNA binding proteins: identification of mitochondrial histones, Molecular BioSystems. 7, 1523-1536.

72. Shu, X., Xiong, X., Song, J., He, C. \& Yi, C. (2016) Base-Resolution Analysis of Cisplatin-DNA Adducts at the Genome Scale, Angewandte Chemie. 55, 14246-14249.

73. Hu, J., Lieb, J. D., Sancar, A. \& Adar, S. (2016) Cisplatin DNA damage and repair maps of the human genome at single-nucleotide resolution, Proc Natl Acad Sci U S A. 113, 11507-11512.

74. Galluzzi, L., Senovilla, L., Vitale, I., Michels, J., Martins, I., Kepp, O., Castedo, M. \& Kroemer, G. (2012) Molecular mechanisms of cisplatin resistance, Oncogene. 31, 1869-83.

75. Chtchigrovsky, M., Eloy, L., Jullien, H., Saker, L., Segal-Bendirdjian, E., Poupon, J., Bombard, S., Cresteil, T., Retailleau, P. \& Marinetti, A. (2013) Antitumor trans-N-heterocyclic carbene-amine-Pt(II) complexes: synthesis of dinuclear species and exploratory investigations of DNA binding and cytotoxicity mechanisms, J Med Chem. 56, 2074-86.

76. Cocetta, V., Ragazzi, E. \& Montopoli, M. (2019) Mitochondrial Involvement in Cisplatin Resistance, International journal of molecular sciences. 20.

77. Wexeslblatt, E., Yavin, E. \& Gibson, D. (2012) Cellular interactions of platinum drugs, Inorg Chim Acta. 393, 75-83.

78. Muoio, D., Berardinelli, F., Leone, S., Coluzzi, E., di Masi, A., Doria, F., Freccero, M., Sgura, A., Folini, M. \& Antoccia, A. (2018) Naphthalene diimide-derivatives G-quadruplex ligands induce cell proliferation inhibition, mild telomeric dysfunction and cell cycle perturbation in U251MG glioma cells, The FEBS journal. 285, 3769-3785.

79. Zheng, X. H., Mu, G., Zhong, Y. F., Zhang, T. P., Cao, Q., Ji, L. N., Zhao, Y. \& Mao, Z. W. (2016)

Trigeminal star-like platinum complexes induce cancer cell senescence through quadruplex-mediated telomere dysfunction, Chem Commun (Camb). 52, 14101-14104.

80. Amato, J., laccarino, N., Pagano, B., Morigi, R., Locatelli, A., Leoni, A., Rambaldi, M., Zizza, P., Biroccio, A., Novellino, E. \& Randazzo, A. (2014) Bis-indole derivatives with antitumor activity turn out to be specific ligands of human telomeric G-quadruplex, Front Chem. 2, 54. 
81. Ourliac-Garnier, I., Poulet, A., Charif, R., Amiard, S., Magdinier, F., Rezaï, K., Gilson, E., GiraudPanis, M. \& Bombard, S. (2010) Platination of telomeric DNA by cisplatin disrupts recognition by TRF2 and TRF1, J Biol Inorg Chem. 15, 641-654.

82. Gomez, D., Paterski, R., Lemarteleur, T., Shin-Ya, K., Mergny, J. L. \& Riou, J. F. (2004) Interaction of telomestatin with the telomeric single-strand overhang, J Biol Chem. 279, 41487-94.

83. Gomez, D., Wenner, T., Brassart, B., Douarre, C., O'Donohue M, F., El Khoury, V., Shin-Ya, K., Morjani, H., Trentesaux, C. \& Riou, J. F. (2006) Telomestatin induced telomere uncapping is modulated by POT1 through G-overhang extension in HT1080 human tumor cells, J Biol Chem. 281, 38721-38729.

84. Huda, N., Tanaka, H., Mendonca, M. S. \& Gilley, D. (2009) DNA damage-induced phosphorylation of TRF2 is required for the fast pathway of DNA double-strand break repair, Mol Cell Biol. 29, 3597604.

85. Rizzo, A., lachettini, S., Salvati, E., Zizza, P., Maresca, C., D'Angelo, C., Benarroch-Popivker, D., Capolupo, A., Del Gaudio, F., Cosconati, S., Di Maro, S., Merlino, F., Novellino, E., Amoreo, C. A., Mottolese, M., Sperduti, I., Gilson, E. \& Biroccio, A. (2017) SIRT6 interacts with TRF2 and promotes its degradation in response to DNA damage, Nucleic Acids Res. 45, 1820-1834.

86. Arnoult, N. \& Karlseder, J. (2015) Complex interactions between the DNA-damage response and mammalian telomeres, Nat Struct Mol Biol. 22, 859-66.

87. Sfeir, A. \& de Lange, T. (2012) Removal of shelterin reveals the telomere end-protection problem, Science. 336, 593-7.

88. Cristini, A., Ricci, G., Britton, S., Salimbeni, S., Huang, S. N., Marinello, J., Calsou, P., Pommier, Y., Favre, G., Capranico, G., Gromak, N. \& Sordet, O. (2019) Dual Processing of R-Loops and Topoisomerase I Induces Transcription-Dependent DNA Double-Strand Breaks, Cell reports. 28, 31673181 e6.

89. Huppert, J. L. \& Balasubramanian, S. (2005) Prevalence of quadruplexes in the human genome, Nucleic Acids Res. 33, 2908-16.

90. Rodriguez, R., Miller, K. M., Forment, J. V., Bradshaw, C. R., Nikan, M., Britton, S., Oelschlaegel, T., Xhemalce, B., Balasubramanian, S. \& Jackson, S. P. (2012) Small-molecule-induced DNA damage identifies alternative DNA structures in human genes, Nat Chem Biol. 8, 301-10.

91. Olivieri, M., Cho, T., Alvarez-Quilon, A., Li, K., Schellenberg, M. J., Zimmermann, M., Hustedt, N., Rossi, S. E., Adam, S., Melo, H., Heijink, A. M., Sastre-Moreno, G., Moatti, N., Szilard, R. K., McEwan, A., Ling, A. K., Serrano-Benitez, A., Ubhi, T., Feng, S., Pawling, J., Delgado-Sainz, I., Ferguson, M. W., Dennis, J. W., Brown, G. W., Cortes-Ledesma, F., Williams, R. S., Martin, A., Xu, D. \& Durocher, D. (2020) A Genetic Map of the Response to DNA Damage in Human Cells, Cell. 182, 481-496 e21. 
92. Yimit, A., Adebali, O., Sancar, A. \& Jiang, Y. (2019) Differential damage and repair of DNA-adducts induced by anti-cancer drug cisplatin across mouse organs, Nat Commun. 10, 309.

93. Wang, D., Hara, R., Singh, G., Sancar, A. \& Lippard, S. J. (2003) Nucleotide excision repair from site-specifically platinum-modified nucleosomes, Biochemistry. 42, 6747-53.

94. Perera, D., Poulos, R. C., Shah, A., Beck, D., Pimanda, J. E. \& Wong, J. W. (2016) Differential DNA repair underlies mutation hotspots at active promoters in cancer genomes, Nature. 532, 259-63. 